ANNALES

POLONICI MATHEMATICI

$96.2(2009)$

\title{
Derivation and well-posedness of Boussinesq/Boussinesq systems for internal waves
}

\author{
by Cung The Anh (Hanoi)
}

\begin{abstract}
We consider the propagation of internal waves at the interface between two layers of immiscrible fluids of different densities, under the rigid lid assumption, with the presence of surface tension and with uneven bottoms. We are interested in the case where the flow has a Boussinesq structure in both the upper and lower fluid domains. Following the global strategy introduced recently by Bona, Lannes and Saut [J. Math. Pures Appl. 89 (2008)], we derive an asymptotic model in this regime, namely the Boussinesq/Boussinesq systems. Then using a contraction-mapping argument and energy methods, we prove that the derived systems which are linearly well-posed are in fact locally nonlinearly well-posed in suitable Sobolev classes. We recover and extend some known results on asymptotic models and well-posedness, for both surface waves and internal waves.
\end{abstract}

\section{Introduction}

Generalities. As shown in $[8,10]$, the propagation of small amplitude long waves on the surface of an ideal fluid (over a horizontal bottom) under the force of gravity is described by the family of Boussinesq systems

$$
\left\{\begin{array}{l}
\left(1-\varepsilon a_{2} \Delta\right) \partial_{t} \zeta+\nabla \cdot V+\varepsilon\left(\nabla \cdot(\zeta V)+a_{1} \Delta \nabla \cdot V\right)=0, \\
\left(1-\varepsilon a_{4} \Delta\right) \partial_{t} V+\nabla \zeta+\varepsilon\left(\frac{1}{2} \nabla|V|^{2}+a_{3} \Delta \nabla \zeta\right)=0,
\end{array}\right.
$$

where $a_{1}, a_{2}, a_{3}, a_{4}$ are defined as follows:

$$
\begin{array}{ll}
a_{1}=\left(\frac{\theta^{2}}{2}-\frac{1}{6}\right) \lambda, & a_{2}=\left(\frac{\theta^{2}}{2}-\frac{1}{6}\right)(1-\lambda), \\
a_{3}=\frac{1-\theta^{2}}{2} \mu, & a_{4}=\frac{1-\theta^{2}}{2}(1-\mu),
\end{array}
$$

and where $0 \leq \theta \leq 1$ and $\lambda, \mu \in \mathbb{R}$ are three parameters. The quantity $\zeta(X, t)+h_{0}, X \in \mathbb{R}^{d}(d=1,2)$, corresponds to the total depth of the liquid

2000 Mathematics Subject Classification: 76B55, 35Q35, 35A07.

Key words and phrases: internal waves, nonflat bottom, surface tension, consistency, Dirichlet-Neumann operator, interface operator, Boussinesq/Boussinesq systems, wellposedness, contraction-mapping argument, energy methods. 
at the point $X$ at time $t$, where $h_{0}$ is undisturbed water depth. The variable $V(X, t)$ represents the horizontal velocity at the point $(X, z)=\left(X, \theta h_{0}\right)$ at time $t$. The Boussinesq approximation is valid when $\varepsilon=a / h_{0} \ll 1$, $\lambda / h_{0} \gg 1$ and the Stokes number $S=a \lambda^{2} / h_{0}^{3}$ is of order 1 , and we have taken $S=1$ when deriving (1.1); here $a$ is the maximum free elevation above the undisturbed level of the fluid of depth $h_{0}$, and $\lambda$ a typical wavelength.

Boussinesq systems have attracted interest because they are fundamental systems describing the motion of small amplitude long waves on the surface of an ideal fluid. Moreover, as seen in [8], one can recover from (1.1) many systems which have already appeared in the literature, such as the classical Boussinesq system, Kaup system, Bona-Smith system, coupled BBM-system, coupled KdV system, coupled KdV-BBM system, coupled BBM-KdV system, etc. The local well-posedness of the Cauchy problem and initial boundary-value problems for Boussinesq type systems has been studied by many authors, including Schonbek [33], Amick [4], Bona and Chen [7], Angulo [5], Kita and Segata [26], Bona, Chen and Saut [9], Fokas and Pelloni [21], and Dougalis, Mitsotakis and Saut [20]. To justify Boussinesq systems, Bona, Colin and Lannes proved in [10] that the solutions of any of the aforementioned systems yield good approximations to the full Euler equations on the long time scale $1 / \varepsilon$. This result was recently extended to the case of nonflat bottoms by Chazel [15]. The justifying of Boussinesq systems for surface waves has now been fully achieved by combining the results of [10], [15] and the large time existence result for the full Euler equation proved very recently by Alvarez-Samaniego and Lannes [1].

Parallel to the theory of surface water waves, the mathematical theory of waves on the interface between two layers of immiscible fluid of different densities has also attracted interest because it is the simplest idealization of internal wave propagation and because of the challenging modelling, mathematical and numerical issues that arise in the analysis of this system. Therefore, in the last decades, it has been studied by many physicists and mathematicians, with regard to both well-posedness [23, 24, 2, 3, 32] and asymptotic models $[16,17,11,19,14,6,29,30]$. A significant step in the theory of internal waves was made in 2008 by Bona, Lannes and Saut [12]. They proposed a general method to derive in a systematic way, and for a large class of scaling regimes, asymptotic models for the propagation of internal waves at the interface between two layers of immiscible fluids of different densities, under the following assumptions: the fluids are ideal, incompressible, irrotational, and under the only influence of gravity, the lids are rigid, the bottom is flat, and there is no surface tension. They derived several classical models and also some new models. They also proved that these asymptotic systems are consistent with the full Euler equations. As they mentioned, these results could be extended to the case of a seabed with structure. 
On the other hand, it is known that surface tension plays an important role in well-posedness of internal waves. In fact, the two-layer water wave system is known to be well-posed in Sobolev spaces in the presence of surface tension $[23,24]$. Therefore, we could take into account an amount of surface tension at the interface between the two homogeneous layers to put oneself in a well-posed situation. Such an approach is used in [30] for the BenjaminOno equation. One interesting question is how the surface tension affects the asymptotic models.

In this paper we are interested in the internal waves problem with nonflat bottom and with the presence of surface tension when the flow has a Boussinesq structure in both the upper and lower fluid domains. The idealized system will be studied, which, when at rest, consists of a homogeneous fluid of depth $d_{1}$ and density $\varrho_{1}$ lying over another homogeneous fluid of mean depth $d_{2}$ and density $\varrho_{2}>\varrho_{1}$. We restrict our study to the case where the bottom can be described by the graph of a function $X \mapsto-d_{1}-d_{2}+b(X)$ and the typical amplitude of the bottom has the same order as that of the interface. Let $a$ be the typical amplitude of the deformation of the interface and $\lambda$ the typical wavelength. We introduce the dimensionless parameters

$$
\gamma:=\frac{\varrho_{1}}{\varrho_{2}}, \quad \delta:=\frac{d_{1}}{d_{2}}, \quad \varepsilon:=\frac{a}{d_{1}}, \quad \mu:=\frac{d_{1}^{2}}{\lambda^{2}}, \quad \varepsilon_{2}:=\frac{a}{d_{2}}=\varepsilon \delta, \quad \mu_{2}:=\frac{d_{2}^{2}}{\lambda^{2}}=\frac{\mu}{\delta^{2}},
$$

the surface tension parameter

$$
\nu:=\frac{\sigma}{\varrho_{2} \lambda^{2}},
$$

and the bottom parameter

$$
\beta:=\frac{b_{0}}{d_{2}} .
$$

Following the global strategy introduced recently by Bona, Lannes and Saut [12] we will prove, when $\varepsilon \sim \mu \sim \varepsilon_{2} \sim \mu_{2} \ll 1$ and in dimensionless variables (see Sect. 2 for details), that the full model is consistent with the following Boussinesq/Boussinesq systems:

$$
\left\{\begin{array}{c}
\left(1-\mu a_{2} \Delta\right) \partial_{t} \zeta+\frac{1}{\gamma+\delta} \nabla \cdot \mathbf{v}_{\alpha}+\varepsilon \frac{\delta^{2}-\gamma}{(\gamma+\delta)^{2}} \nabla \cdot\left(\zeta \mathbf{v}_{\alpha}\right) \\
-\frac{\varepsilon \delta^{2}}{(\gamma+\delta)^{2}} \nabla \cdot\left(b \mathbf{v}_{\alpha}\right)+\mu a_{1} \nabla \cdot \Delta \mathbf{v}_{\alpha}=0, \\
\left(1-\mu a_{4} \Delta\right) \partial_{t} \mathbf{v}_{\alpha}+(1-\gamma) \nabla \zeta+\frac{\varepsilon}{2} \frac{\delta^{2}-\gamma}{(\gamma+\delta)^{2}} \nabla\left|\mathbf{v}_{\alpha}\right|^{2} \\
+\mu a_{3}(1-\gamma) \Delta \nabla \zeta-\varepsilon \sqrt{\mu} \nu \Delta \nabla \zeta=0,
\end{array}\right.
$$

where $\zeta$ is the surface elevation, $\mathbf{v}_{\alpha}=(1-\mu \alpha \Delta)^{-1} \mathbf{v}$ with $\mathbf{v}$ the "velocity variable" (see Sect. 2.1 for definition), and the constants $a_{1}, a_{2}, a_{3}, a_{4}$ are given by 


$$
\begin{aligned}
& a_{1}=\frac{\left(1-\alpha_{1}\right)(1+\gamma \delta)-3 \delta \alpha(\gamma+\delta)}{3 \delta(\gamma+\delta)^{2}}, \quad a_{2}=\frac{\gamma \alpha_{1}}{3(\gamma+\delta)}, \\
& a_{3}=\alpha \alpha_{2}, \quad a_{4}=\alpha\left(1-\alpha_{2}\right) .
\end{aligned}
$$

It is worth noticing that when $b=0$ and $\nu=0$ we recover the Boussinesq/Boussinesq systems for internal waves in the case of a flat bottom and absence of surface tension derived in [12], and that the Boussinesq system for surface waves in the absence of surface tension is formally recovered by taking $\gamma=0, \delta=1, \nu=0$ (and $b=0$ if the bottom is flat). The dispersion relation associated to $(1.2)$ is

$$
\omega^{2}=|\mathbf{k}|^{2} \frac{\left(\frac{1}{\gamma+\delta}-\mu a_{1}|\mathbf{k}|^{2}\right)\left[(1-\gamma)\left(1-\mu a_{3}|\mathbf{k}|^{2}\right)+\varepsilon \sqrt{\mu} \nu|\mathbf{k}|^{2}\right]}{\left(1+\mu a_{2}|\mathbf{k}|^{2}\right)\left(1+\mu a_{4}|\mathbf{k}|^{2}\right)} .
$$

It follows that (1.2) is linearly well-posed when $a_{2}, a_{4} \geq 0$ and $a_{1} \leq 0$, $a_{3} \leq \varepsilon \nu /((1-\gamma) \sqrt{\mu})$. Following the general lines of the analogous proof of [9], we will show that the Boussinesq/Boussinesq systems (1.2) which are linearly well-posed are in fact locally nonlinearly well-posed in suitable Sobolev classes. When $a_{2}, a_{4}>0$ we will prove well-posedness by realizing the systems as systems coupled only through nonlinear terms and using a contraction-mapping argument. The other systems (i.e. when $a_{2}=0$ or $a_{4}=0$ ) are analysed using energy methods.

In this paper we shall focus on the case $d=2$ (i.e. when $X=(x, y)$ ) because it is more complicated. However, the method works for both one and two dimensions and the results in one dimension are better than the ones in the case of dimension two as far as the assumed level of regularity of the initial data is concerned. This is explained in Remarks 3.2 and 3.3 below. It is worth noticing that when $\gamma=0, \delta=1$, we recover the Boussinesq systems for surface waves, so we also obtain well-posedness results for them. In particular, we extend the results of [9] to two dimensions and to the case with surface tension. It is also noticed that in the presence of surface tension, the class of Boussinesq/Boussinesq systems that are linearly and/or nonlinearly well-posed is larger than the corresponding one in the absence of surface tension. Thus, the surface tension makes the well-posedness results better, as for the full Euler equation [24].

Notations. Denote by $X$ the $d$-dimensional horizontal variable $(d=1,2)$, and by $z$ the vertical variable. For $\mu>0$, we introduce scaled versions of the gradient and Laplace operators, namely $\nabla_{X, z}^{\mu}=\left(\sqrt{\mu} \nabla^{T}, \partial_{z}\right)^{T}$ and $\Delta_{X, z}^{\mu}=\nabla_{X, z}^{\mu} \cdot \nabla_{X, z}^{\mu}=\mu \Delta_{X}+\partial_{z}^{2}$. We use two Fourier multipliers $\mathbf{T}_{\mu}$ and $\mathbf{T}_{\mu_{2}}$ defined as follows:

$$
\mathbf{T}_{\mu}=\tanh (\sqrt{\mu}|D|), \quad \mathbf{T}_{\mu_{2}}=\tanh \left(\sqrt{\mu_{2}}|D|\right),
$$

where $|D|=(-\Delta)^{1 / 2}$. The orthogonal projector onto the gradient vector 
fields of $L^{2}\left(\mathbb{R}^{d}\right)^{d}$ is written $\Pi$ and is defined by the formula

$$
\Pi=-\frac{\nabla \nabla^{T}}{|D|^{2}} .
$$

The $L^{p}=L^{p}\left(\mathbb{R}^{2}\right)$-norm will be denoted by $|\cdot| p$ for $1 \leq p \leq \infty$, and the $H^{s}=H^{s}\left(\mathbb{R}^{2}\right)$-norm by $\|\cdot\|_{s}$ or $|\cdot|_{H^{s}}$. Note that the $L^{2}$-norm of a function $f$ is written both as $|f|_{2}$ and $\|f\|_{0}$. The product space $X \times X$ will sometimes be abbreviated as $X^{2}$, and a function $f=\left(f_{1}, f_{2}\right)$ in $X^{2}$ carries the norm

$$
\|f\|_{X^{2}}=\left(\left\|f_{1}\right\|^{2}+\left\|f_{2}\right\|^{2}\right)^{1 / 2} .
$$

For convenience, we also use the notations $\|\cdot\|_{s}$ and $|\cdot|_{p}$ for a vector function $f$. Similarly, $\langle\cdot, \cdot\rangle$ denotes the inner products in both $L^{2}\left(\mathbb{R}^{2}\right)$ and $L^{2}\left(\mathbb{R}^{2}\right)^{2}$. We use the Bessel potential $\Lambda^{s}:=(1-\Delta)^{s / 2}, s \in \mathbb{R}$. Note that $H^{s}\left(\mathbb{R}^{2}\right)=\Lambda^{-s} L^{2}\left(\mathbb{R}^{2}\right)$ and thus $\|u\|_{s}=\left\|\Lambda^{s} u\right\|_{0}$ for $u \in H^{s}\left(\mathbb{R}^{2}\right)$. If $f$ and $u$ are two functions defined on $\mathbb{R}^{2}$, we use the Fourier multiplier notation $f(D) u$ which is defined in terms of Fourier transforms:

$$
\widehat{f(D) u}=f \hat{u} .
$$

We denote by $[A, B]:=A B-B A$ the commutator of the operators $A, B$. Thus, $\left[\Lambda^{s}, f\right] g=\Lambda^{s}(f g)-f \Lambda^{s} g$, where $f$ is regarded as a multiplication operator.

We use $C$ to denote various constants whose values may change with each appearance, and use the generic notation $C\left(\lambda_{1}, \lambda_{2}, \ldots\right)$ to denote a nondecreasing function of the parameters $\lambda_{1}, \lambda_{2}, \ldots$.

Organization of the paper. The plan of this paper is as follows. In Section 2, when the flow has a Boussinesq structure in both the upper and lower fluid domains, i.e. when $\varepsilon \sim \mu \sim \varepsilon_{2} \sim \mu_{2} \ll 1$, we derive the Boussinesq/Boussinesq systems (1.2) from the full model. To do this, we first derive asymptotic expansions of the Dirichlet-Neumann operator $\mathbf{G}^{\mu}[\varepsilon \zeta]$ and the interface operator $\mathbf{H}^{\mu, \delta}[\varepsilon \zeta, \beta b]$ in this regime. Then, using the expansions of these two operators, a change of variables and a suitable "BBM trick", we get the Boussinesq/Boussinesq systems depending on three parameters. We also prove that the systems derived are consistent with the full Euler system. In Section 3, we study the local well-posedness of the Cauchy problem for the Boussinesq/Boussinesq systems by using a contraction-mapping argument and energy methods.

2. Model systems. In this section we derive an asymptotic model for internal waves when the flow has a Boussinesq structure in both the upper and lower fluid domains. In order to study the influence of surface tension and bottom topography on the asymptotic model, we introduce two new parameters, one characterizing the shape of the bottom and 
the other characterizing the effect of surface tension. We follow here the same strategy as in $[8,10,15]$ for surface waves, and as in [12] for internal waves. Namely, we rewrite the full system as a system of two evolution equations on $\mathbb{R}^{d}(d=1,2)$. The reformulated system, which depends only upon the spatial variable on the interface, involves two nonlocal operators, the Dirichlet-Neumann operator $\mathbf{G}[\zeta]$ and the interface operator $\mathbf{H}[\zeta, b]$ (the latter appears only in the internal waves theory). The asymptotic model is obtained by expanding the two nonlocal operators with respect to suitable small parameters that depend variously on the amplitude, wavelengths and depth ratio of the two layers, with order higher than the orders of the bottom terms and of the term containing the surface tension. Then a family of asymptotic models may be inferred by using a "BBM trick" and a suitable change of the dependent variables. The freedom to choose parameters in order to make the corresponding system linearly well-posed is just one of the advantages of having families of systems. The consistency of this model with the full Euler equations is also established.

2.1. The Euler equations and the full model. We consider the internal waves problem in the case of uneven bottoms and with the presence of surface tension. The idealized system that will be studied here, when at rest, consists of a homogeneous fluid of depth $d_{1}$ and density $\varrho_{1}$ lying over another homogeneous fluid of mean depth $d_{2}$ and density $\varrho_{2}>\varrho_{1}$. We restrict our study to the case where the bottom can be described by the graph of a function $X \mapsto-d_{1}-d_{2}+b(X)$ and the typical amplitude of the bottom has the same order as that of the interface. Let $\Omega_{t}^{i}$ be the region occupied by fluid $i$ at time $t(i=1,2), \Gamma_{1}:=\{z=0\}$ and $\Gamma_{2}:=\left\{z=-d_{1}-d_{2}+b(X)\right\}$ be two rigid surfaces, and $\Gamma_{t}:=\left\{z=-d_{1}+\zeta(t, X)\right\}$ be the interface between the fluids. As shown in [12], the motion of the internal wave, under Bernoulli's formulation, in terms of two velocity potentials $\Phi_{1}, \Phi_{2}$, is described by the following system of equations:

$$
\begin{aligned}
& \Delta_{X, z} \Phi_{i}=0 \quad \text { in } \Omega_{t}^{i}, \\
& \partial_{t} \Phi_{i}+\frac{1}{2}\left|\nabla_{X, z} \Phi_{i}\right|^{2}=-\frac{P_{i}}{\varrho_{i}}-g z \text { in } \Omega_{t}^{i}, \\
& \partial_{z} \Phi_{1}=0 \quad \text { on } \Gamma_{1}, \quad \partial_{\mathbf{n}^{-}} \Phi_{2}=0 \quad \text { on } \Gamma_{2}, \\
& \partial_{t} \zeta=\sqrt{1+|\nabla \zeta|^{2}} \partial_{n} \Phi_{1} \quad \text { on } \Gamma_{t}, \\
& \partial_{\mathbf{n}^{+}} \Phi_{1}=\partial_{\mathbf{n}^{+}} \Phi_{2} \text { on } \Gamma_{t}, \\
& P_{1}-P_{2}=\sigma \nabla \cdot\left(\frac{\nabla \zeta}{\sqrt{1+|\nabla \zeta|^{2}}}\right),
\end{aligned}
$$


where $P_{1}$ and $P_{2}$ are the pressures inside the upper and lower fluids, $g$ is the acceleration of gravity, $\mathbf{n}^{+}:=(-\nabla \zeta, 1)^{T} / \sqrt{1+|\nabla \zeta|^{2}}$ denotes the outward normal vector to the interface, $\mathbf{n}^{-}:=(\nabla b,-1)^{T} / \sqrt{1+|\nabla b|^{2}}$ the outward normal vector to the bottom, $\partial_{\mathbf{n}}:=\mathbf{n} . \nabla_{X, z}$, and $\sigma=$ const $>0$ is the surface tension coefficient.

Introduce the trace of the potentials $\Phi_{1}$ and $\Phi_{2}$ at the interface,

$$
\psi_{i}(t, X):=\Phi_{i}\left(t, X,-d_{1}+\zeta(t, X)\right), \quad i=1,2 .
$$

One can evaluate (2.2) at the interface and use (2.4) and (2.5) to obtain a set of equations coupling $\zeta$ to $\psi_{i}$ :

$$
\begin{gathered}
\partial_{t} \zeta-\sqrt{1+|\nabla \zeta|^{2}} \partial_{n} \Phi_{i}=0 \\
\varrho_{i}\left(\partial_{t} \psi_{i}+g \zeta+\frac{1}{2}\left|\nabla \psi_{i}\right|^{2}-\frac{\left(\sqrt{1+|\nabla \zeta|^{2}}\left(\partial_{n} \Phi_{i}\right)+\nabla \zeta . \nabla \psi_{i}\right)^{2}}{2\left(1+|\nabla \zeta|^{2}\right)^{2}}\right)=-P_{i},
\end{gathered}
$$

where $\partial_{n} \Phi_{i}$ and $P_{i}$ are both evaluated at the interface $z=-d_{1}+\zeta(t, X)$. As in [12], by using two local operators, the Dirichlet-Neumann operator $\mathbf{G}[\zeta]$ and the interface operator $\mathbf{H}[\zeta, b]$, defined by

$$
\mathbf{G}[\zeta] \psi_{1}=\sqrt{1+|\nabla \zeta|^{2}}\left(\partial_{n} \Phi_{1}\right)_{\mid z=-d_{1}+\zeta}, \quad \mathbf{H}[\zeta, b] \psi_{1}=\nabla \psi_{2},
$$

and using (2.6), we get

$$
\begin{aligned}
\partial_{t}\left(\psi_{2}-\gamma \psi_{1}\right)+g(1-\gamma) \zeta+\frac{1}{2}\left(\left|\mathbf{H}[\zeta, b] \psi_{1}\right|^{2}-\gamma\left|\nabla \psi_{1}\right|^{2}\right) & \\
& +\mathcal{N}\left(\zeta, b, \psi_{1}\right)-\frac{\sigma}{\varrho_{2}} K(\zeta)=0,
\end{aligned}
$$

where $\gamma=\varrho_{1} / \varrho_{2}, K(\zeta)=\nabla \cdot\left(\nabla \zeta / \sqrt{1+|\nabla \zeta|^{2}}\right)$ and

$$
\mathcal{N}\left(\zeta, b, \psi_{1}\right):=\frac{\gamma\left(\mathbf{G}[\zeta] \psi_{1}+\nabla \zeta . \nabla \psi_{1}\right)^{2}-\left(\mathbf{G}[\zeta] \psi_{1}+\nabla \zeta . \mathbf{H}[\zeta, b] \psi_{1}\right)^{2}}{2\left(1+|\nabla \zeta|^{2}\right)^{2}} .
$$

Taking the gradient of this equation and using (2.7) then gives the system of equations

$$
\left\{\begin{array}{l}
\partial_{t} \zeta-\mathbf{G}[\zeta] \psi_{1}=0 \\
\partial_{t}\left(\mathbf{H}[\zeta, b] \psi_{1}-\gamma \nabla \psi_{1}\right)+g(1-\gamma) \nabla \zeta \\
\quad+\frac{1}{2} \nabla\left(\left|\mathbf{H}[\zeta, b] \psi_{1}\right|^{2}-\gamma\left|\nabla \psi_{1}\right|^{2}\right)+\nabla \mathcal{N}\left(\zeta, b, \psi_{1}\right)-\frac{\sigma}{\varrho_{2}} \nabla K(\zeta)=0
\end{array}\right.
$$

for $\zeta$ and $\psi_{1}$.

The asymptotic expansion of (2.9) is more transparent when these equations are written in dimensionless variables. Denoting by $a$ a typical amplitude of the deformation of the interface in question, and by $\lambda$ a typical wavelength, we introduce the dimensionless indendent variables

$$
\tilde{X}:=\frac{X}{\lambda}, \quad \tilde{z}:=\frac{z}{d_{1}}, \quad \tilde{t}:=\frac{t}{\lambda / \sqrt{g d_{1}}} .
$$


Likewise, we define the dimensionless functions

$$
\tilde{\zeta}:=\frac{\zeta}{a}, \quad \tilde{\psi}_{1}:=\frac{\psi_{1}}{a \lambda \sqrt{g / d_{1}}}, \quad \tilde{b}=\frac{b}{b_{0}},
$$

as well as the dimensionless parameters

$$
\gamma:=\frac{\varrho_{1}}{\varrho_{2}}, \quad \delta:=\frac{d_{1}}{d_{2}}, \quad \varepsilon:=\frac{a}{d_{1}}, \quad \mu:=\frac{d_{1}^{2}}{\lambda^{2}}, \quad \beta:=\frac{b_{0}}{d_{2}}, \quad \nu:=\frac{\sigma}{\varrho_{2} \lambda^{2}} .
$$

Here $b_{0}$ is the typical amplitude of the bottom and therefore $\beta$ characterizes the shape of the bottom, and $\nu$ is the surface tension parameter. It is also notationally convenient to introduce two other (redundant) parameters $\varepsilon_{2}$ and $\mu_{2}$ defined as

$$
\varepsilon_{2}:=\frac{a}{d_{2}}=\varepsilon \delta, \quad \mu_{2}:=\frac{d_{2}^{2}}{\lambda^{2}}=\frac{\mu}{\delta^{2}} .
$$

In dimensionless variables, the upper fluid domain $\Omega_{1}$ and the lower fluid domain $\Omega_{2}$ have the forms

$$
\begin{aligned}
& \Omega_{1}=\left\{(X, z) \in \mathbb{R}^{d+1}:-1+\varepsilon \zeta<z<0\right\}, \\
& \Omega_{2}=\left\{(X, z) \in \mathbb{R}^{d+1}:-1-\frac{1}{\delta}+\frac{1}{\delta} \beta b(X)<z<-1+\varepsilon \zeta\right\} .
\end{aligned}
$$

In order to prevent the interface from touching the horizontal boundaries, we assume that there are strict positive constants $H_{1}$ and $H_{2}$ such that

$$
\begin{aligned}
1-\varepsilon \zeta \geq H_{1} & \text { on } \mathbb{R}^{d}, \\
1+\varepsilon \delta \zeta-\beta b(X) \geq H_{2} & \text { on } \mathbb{R}^{d} .
\end{aligned}
$$

To write (2.9) in dimensionless variables, following [12] we introduce the definitions of the dimensionless Dirichlet-Neumann operator $\mathbf{G}^{\mu}[\varepsilon \zeta]$ and the dimensionless interface operator $\mathbf{H}^{\mu, \delta}[\varepsilon \zeta, \beta b]$.

Definition 1. Let $\zeta \in W^{2, \infty}\left(\mathbb{R}^{d}\right)$ be such that (2.10) is satisfied and let $\psi_{1} \in H^{3 / 2}\left(\mathbb{R}^{d}\right)$. If $\Phi_{1}$ is the unique solution in $H^{2}\left(\Omega_{1}\right)$ of the boundary value problem

$$
\left\{\begin{array}{l}
\mu \Delta \Phi_{1}+\partial_{z}^{2} \Phi_{1}=0 \quad \text { in } \Omega_{1}, \\
\left.\partial_{z} \Phi_{1}\right|_{z=0}=0,\left.\quad \Phi_{1}\right|_{z=-1+\varepsilon \zeta(X)}=\psi_{1},
\end{array}\right.
$$

then $\mathbf{G}^{\mu}[\varepsilon \zeta] \psi_{1} \in H^{1 / 2}\left(\mathbb{R}^{d}\right)$ is defined by

$$
\begin{aligned}
\mathbf{G}^{\mu}[\varepsilon \zeta] \psi_{1} & =-\mu \varepsilon \nabla \zeta .\left.\nabla \Phi_{1}\right|_{z=-1+\varepsilon \zeta(X)}+\left.\partial_{z} \Phi_{1}\right|_{z=-1+\varepsilon \zeta(X)} \\
& =\left.\sqrt{1+\varepsilon^{2}|\nabla \zeta|^{2}} \partial_{n} \Phi_{1}\right|_{z=-1+\varepsilon \zeta(X)},
\end{aligned}
$$

where $\left.\partial_{n} \Phi_{1}\right|_{z=-1+\varepsilon \zeta(X)}$ stands for the upper conormal derivative associated to the elliptic operator $\mu \Delta \Phi_{1}+\partial_{z}^{2} \Phi_{1}$. 
Definition 2. Let $\zeta \in W^{2, \infty}\left(\mathbb{R}^{d}\right)$ and $b \in W^{2, \infty}\left(\mathbb{R}^{d}\right)$ be such that $(2.10)$ and (2.11) are satisfied and let $\psi_{1} \in H^{3 / 2}\left(\mathbb{R}^{d}\right)$. If $\Phi_{2}$ is the unique solution in $H^{2}\left(\Omega_{2}\right)$ (up to a constant) of the boundary value problem

$$
\left\{\begin{array}{l}
\mu \Delta \Phi_{2}+\partial_{z}^{2} \Phi_{2}=0 \quad \text { in } \Omega_{2} \\
\left.\partial_{n} \Phi_{2}\right|_{z=-1-\frac{1}{\delta}+\frac{1}{\delta} \beta b(X)}=0, \\
\left.\partial_{n} \Phi_{2}\right|_{z=-1+\varepsilon \zeta(X)}=\frac{1}{\sqrt{1+\varepsilon^{2}|\nabla \zeta|^{2}}} \mathbf{G}^{\mu}[\varepsilon \zeta] \psi_{1},
\end{array}\right.
$$

then $\mathbf{H}^{\mu, \delta}[\varepsilon \zeta, \beta b] \psi_{1} \in H^{1 / 2}\left(\mathbb{R}^{d}\right)$ is defined by

$$
\mathbf{H}^{\mu, \delta}[\varepsilon \zeta, \beta b] \psi_{1}=\nabla\left(\left.\Phi_{2}\right|_{z=-1+\varepsilon \zeta(X)}\right) .
$$

The equation (2.9) can therefore be written in dimensionless variables as

$$
\left\{\begin{array}{c}
\partial_{\tilde{t}} \tilde{\zeta}-\frac{1}{\mu} \mathbf{G}^{\mu}[\varepsilon \tilde{\zeta}] \tilde{\psi_{1}}=0 \\
\partial_{\tilde{t}}\left(\mathbf{H}^{\mu, \delta}[\varepsilon \tilde{\zeta}, \beta \tilde{b}] \tilde{\psi}_{1}-\gamma \nabla \tilde{\psi}_{1}\right)+(1-\gamma) \nabla \tilde{\zeta} \\
\quad+\frac{\varepsilon}{2} \nabla\left(\left|\mathbf{H}^{\mu, \delta}[\varepsilon \tilde{\zeta}, \beta \tilde{b}] \tilde{\psi}_{1}\right|^{2}-\gamma\left|\nabla \tilde{\psi}_{1}\right|^{2}\right) \\
\quad+\varepsilon \nabla \mathcal{N}^{\mu, \delta}\left(\varepsilon \tilde{\zeta}, \beta \tilde{b}, \tilde{\psi}_{1}\right)-\frac{\sigma}{\varrho_{2}} \nabla K(\tilde{\zeta})=0
\end{array}\right.
$$

where

$$
\begin{aligned}
\mathcal{N}^{\mu, \delta} & \left(\zeta, b, \psi_{1}\right) \\
& :=\mu \frac{\gamma\left(\frac{1}{\mu} \mathbf{G}^{\mu}[\zeta] \psi_{1}+\nabla \zeta . \nabla \psi_{1}\right)^{2}-\left(\frac{1}{\mu} \mathbf{G}^{\mu}[\zeta] \psi_{1}+\nabla \zeta . \mathbf{H}^{\mu, \delta}[\zeta, b] \psi_{1}\right)^{2}}{2\left(1+\mu|\nabla \zeta|^{2}\right)^{2}} .
\end{aligned}
$$

The tildes which indicate the nondimensional quantities will be systematically dropped henceforth.

We will propose a model system of equations for the internal waves in the Boussinesq/Boussinesq regime $\varepsilon \sim \mu \sim \varepsilon_{2} \sim \mu_{2} \ll 1$ by obtaining the asymptotic form of the equations (2.14) in this regime. The asymptotic model is a $(1+d)$-dimensional system coupling the surface elevation $\zeta$ to the "velocity variable" $\mathbf{v}$ defined to be

$$
\mathbf{v}:=\mathbf{H}^{\mu, \delta}[\varepsilon \zeta, \beta b] \psi_{1}-\gamma \nabla \psi_{1} .
$$

(For the surface water-wave problem formally recovered by taking $\gamma=0$ and $\delta=1, \mathbf{v}$ is the horizontal velocity evaluated at the free surface). It will be rigorously established that the internal wave equations (2.14) are consistent with the asymptotic model for $(\zeta, \mathbf{v})$ derived in this paper in the following precise sense.

Definition 3. The internal wave equations (2.14) are consistent with a system $S$ of $d+1$ equations for $\zeta$ and $\mathbf{v}$ if for all sufficiently smooth solutions $\left(\zeta, \psi_{1}\right)$ of $(2.14)$ such that $(2.10)$ and (2.11) are satisfied, the pair 
$\left(\zeta, \mathbf{v}=\mathbf{H}^{\mu, \delta}[\varepsilon \zeta, \beta b] \psi_{1}-\gamma \nabla \psi_{1}\right)$ solves $S$ up to a small residual called the precision of the asymptotic model.

2.2. Asymptotic expansions of the operators. First, from Lemma 1 and Remark 11 in [12] we have the asymptotic expansion of the DirichletNeumann operator $\mathbf{G}^{\mu}[\varepsilon \zeta]$ which we will use in this paper.

Proposition 2.1. Let $s>d / 2$ and $\zeta \in H^{s+3 / 2}\left(\mathbb{R}^{d}\right)$ be such that (2.10) is satisfied. Then for all $\mu \in(0,1)$ and $\psi$ such that $\nabla \psi \in H^{s+5 / 2}\left(\mathbb{R}^{d}\right)$, one has

$$
\begin{aligned}
\mid \mathbf{G}^{\mu}[\varepsilon \zeta] \psi-\left(\mu \nabla \cdot((1-\varepsilon \zeta) \nabla \psi)+\frac{\mu^{2}}{3} \nabla\right. & . \Delta \nabla \psi)\left.\right|_{H^{s}} \\
& \leq \mu^{3} C\left(|\zeta|_{H^{s+3 / 2}},|\nabla \psi|_{H^{s+5 / 2}}\right),
\end{aligned}
$$

uniformly with respect to $\varepsilon \in[0,1]$.

Attention is now turned to the interface operator $\mathbf{H}^{\mu, \delta}[\varepsilon \zeta, \beta b]$. As already mentioned, in this paper we restrict our study to the case where the amplitude $b_{0}$ of the bottom has the same order as the typical amplitude $a$ of the interface. Noting that $\varepsilon_{2}=a / d_{2}$ and $\beta=b_{0} / d_{2}$, we therefore have $\beta=O\left(\varepsilon_{2}\right)$. Since $\varepsilon_{2} \ll 1$ in the Boussinesq/Boussinesq regime, the condition $\beta=O\left(\varepsilon_{2}\right)$ means that we consider bottoms with small variations in the amplitude. This restriction enables us to use the WKB method to derive the asymptotic expansion of the interface operator $\mathbf{H}^{\mu, \delta}[\varepsilon \zeta, \beta b]$. In order to improve readability, we will write $\beta=\varepsilon_{2}$ for this regime.

The boundary value problem (2.13) plays an important role in the analysis of the operator $\mathbf{H}^{\mu, \delta}[\varepsilon \zeta, \beta b]$. We first transform the problem (2.13) into a variable-coefficient, boundary value problem on the flat strip $\mathbf{S}=$ $\mathbb{R}^{d} \times(-1,0)$ by using the diffeomorphism

$$
\sigma: \mathbf{S} \rightarrow \Omega_{2}, \quad(X, z) \mapsto \sigma(X, z)=\left(X,(1+\varepsilon \delta \zeta-\beta b(X)) \frac{z}{\delta}+(-1+\varepsilon \zeta)\right) .
$$

By Proposition 2.7 in [27], noticing that $\beta=\varepsilon_{2}$, one can see that $\Phi_{2}$ solves (2.13) if and only if $\underline{\Phi}_{2}:=\Phi_{2} \circ \sigma$ solves

$$
\left\{\begin{array}{l}
\nabla_{X, z}^{\mu_{2}} \cdot Q^{\mu_{2}}\left[\varepsilon_{2} \zeta, \varepsilon_{2} b\right] \nabla_{X, z}^{\mu_{2}} \underline{\Phi}_{2}=0 \quad \text { in } \mathbf{S}, \\
\partial_{n} \underline{\Phi}_{\left.2\right|_{z=0}}=\frac{1}{\delta} \mathbf{G}^{\mu}[\varepsilon \zeta] \psi_{1},\left.\quad \partial_{n} \underline{\Phi}_{2}\right|_{z=-1}=0,
\end{array}\right.
$$

with

$$
\begin{aligned}
Q^{\mu_{2}}\left[\varepsilon_{2} \zeta, \varepsilon_{2} b\right] & \\
& =\left(\begin{array}{cc}
{\left[1+\varepsilon_{2}(\zeta-b)\right] I_{d \times d}} & -\sqrt{\mu_{2}} \varepsilon_{2}[(z+1) \nabla \zeta-z \nabla b] \\
-\sqrt{\mu_{2}} \varepsilon_{2}[(z+1) \nabla \zeta-z \nabla b]^{T} & \frac{1+\mu_{2} \varepsilon_{2}^{2}|(z+1) \nabla \zeta-z \nabla b|^{2}}{1+\varepsilon_{2}(\zeta-b)}
\end{array}\right) .
\end{aligned}
$$


Then an asymptotic expansion of

$$
\mathbf{H}^{\mu, \delta}[\varepsilon \zeta, \beta b] \psi_{1}=\nabla\left(\left.\underline{\Phi}_{2}\right|_{z=0}\right)
$$

is obtained by finding an approximation $\underline{\Phi}_{\text {app }}$ to the solution of (2.16) and by using the formal relationship $\mathbf{H}^{\mu, \delta}[\varepsilon \zeta, \beta b] \psi_{1} \sim \nabla\left(\left.\underline{\Phi}_{\text {app }}\right|_{z=0}\right)$ thanks to Proposition 3 in [12]. This procedure is justified rigorously in Proposition 2.2 below (see also the proof of Corollary 1 in [12]).

We now find an approximate solution $\underline{\Phi}_{\text {app }}$ to (2.16). We decompose the matrix $Q^{\mu_{2}}\left[\varepsilon_{2} \zeta, \varepsilon_{2} b\right]$ as follows:

$$
Q^{\mu_{2}}\left[\varepsilon_{2} \zeta, \varepsilon_{2} b\right]=Q_{0}+\varepsilon_{2} Q_{1}+\varepsilon_{2}^{2} Q_{2},
$$

where

$$
\begin{aligned}
Q_{0} & =\left(\begin{array}{cc}
I_{d \times d} & 0 \\
0 & 1
\end{array}\right), \\
Q_{1} & =\left(\begin{array}{cc}
(\zeta-b) I_{d \times d} & -\sqrt{\mu_{2}}[(z+1) \nabla \zeta-z \nabla b] \\
-\sqrt{\mu_{2}}[(z+1) \nabla \zeta-z \nabla b]^{T} & -(\zeta-b)
\end{array}\right), \\
Q_{2} & =\left(\begin{array}{cc}
0 & 0 \\
0 & \frac{(\zeta-b)^{2}+\mu_{2}|(z+1) \nabla \zeta-z \nabla b|^{2}}{1+\varepsilon_{2}(\zeta-b)}
\end{array}\right),
\end{aligned}
$$

and construct $\underline{\Phi}_{\text {app }}$ having the form

$$
\underline{\Phi}_{\mathrm{app}}=\Phi^{(0)}+\varepsilon_{2} \Phi^{(1)} .
$$

We have

$$
\begin{aligned}
\nabla_{X, z}^{\mu_{2}} \cdot Q^{\mu_{2}}\left[\varepsilon_{2} \zeta\right. & \left., \varepsilon_{2} b\right] \nabla_{X, z}^{\mu_{2}} \underline{\Phi}_{\mathrm{app}} \\
& =\nabla_{X, z}^{\mu_{2}} \cdot\left(Q_{0}+\varepsilon_{2} Q_{1}+\varepsilon_{2}^{2} Q_{2}\right) \nabla_{X, z}^{\mu_{2}}\left(\Phi^{(0)}+\varepsilon_{2} \Phi^{(1)}\right) \\
& =\Delta_{X, z}^{\mu_{2}} \Phi^{(0)}+\varepsilon_{2}\left(\Delta_{X, z}^{\mu_{2}} \Phi^{(1)}+\nabla_{X, z}^{\mu_{2}} \cdot Q_{1} \nabla_{X, z}^{\mu_{2}} \Phi^{(0)}\right)+O\left(\varepsilon_{2}^{2}\right),
\end{aligned}
$$

and at $z=0$ and $z=-1$,

$$
\begin{aligned}
\partial_{n} \underline{\Phi}_{\mathrm{app}} & =\mathbf{e}_{z} \cdot Q^{\mu_{2}}\left[\varepsilon_{2} \zeta, \varepsilon_{2} b\right] \nabla_{X, z}^{\mu_{2}} \underline{\Phi}_{\mathrm{app}} \\
& =\mathbf{e}_{z} \cdot\left(Q_{0}+\varepsilon_{2} Q_{1}+\varepsilon_{2}^{2} Q_{2}\right) \nabla_{X, z}^{\mu_{2}}\left(\Phi^{(0)}+\varepsilon_{2} \Phi^{(1)}\right) \\
& =\partial_{z} \Phi^{(0)}+\varepsilon_{2}\left(\partial_{z} \Phi^{(1)}+\mathbf{e}_{z} \cdot Q_{1} \nabla_{X, z}^{\mu_{2}} \Phi^{(0)}\right)+O\left(\varepsilon_{2}^{2}\right) .
\end{aligned}
$$

Since it is known from Proposition 2.1 that

$$
\frac{1}{\delta} \mathbf{G}^{\mu}[\varepsilon \zeta] \psi_{1}=\frac{\mu}{\delta} \nabla \cdot\left(h_{1} \nabla \psi_{1}\right)+\frac{\mu^{2}}{3 \delta} \nabla \cdot\left(\Delta \nabla \psi_{1}\right)+O\left(\frac{\mu^{3}}{\delta}\right), \quad \text { where } h_{1}=1-\varepsilon \zeta,
$$

one deduces that $\underline{\Phi}_{\text {app }}$ solves (2.16) up to order $O\left(\varepsilon_{2}^{2}+\mu^{3} / \delta\right)$ provided that 
$\Phi^{(0)}$ and $\Phi^{(1)}$ solve the following boundary value problems:

$$
\begin{aligned}
& \left\{\begin{array}{l}
\Delta_{X, z}^{\mu_{2}} \Phi^{(0)}=0, \\
\left.\partial_{z} \Phi^{(0)}\right|_{z=0}=\frac{\mu}{\delta} \nabla \cdot\left(h_{1} \nabla \psi_{1}\right),\left.\quad \partial_{z} \Phi^{(0)}\right|_{z=-1}=0,
\end{array}\right. \\
& \left\{\begin{array}{l}
\Delta_{X, z}^{\mu_{2}} \Phi^{(1)}=-\nabla_{X, z}^{\mu_{2}} \cdot Q_{1} \nabla_{X, z}^{\mu_{2}} \Phi^{(0)}, \\
\left.\partial_{z} \Phi^{(1)}\right|_{z=0}=\frac{\mu^{2}}{3 \delta} \nabla \cdot\left(\Delta \nabla \psi_{1}\right)-\left.\mathbf{e}_{z} \cdot Q_{1} \nabla_{X, z}^{\mu_{2}} \Phi^{(0)}\right|_{z=0}, \\
\left.\partial_{z} \Phi^{(1)}\right|_{z=-1}=-\mathbf{e}_{z} \cdot Q_{1} \nabla_{X, z}^{\mu_{2}} \Phi_{\mid z=-1}^{(0)} \cdot
\end{array}\right.
\end{aligned}
$$

First, it is easy to find that

$$
\Phi^{(0)}(X, z)=\sqrt{\mu} \frac{\cosh \left(\sqrt{\mu_{2}}(z+1)|D|\right)}{\cosh \left(\sqrt{\mu_{2}}|D|\right)} \frac{1}{|D| \tanh \left(\sqrt{\mu_{2}}|D|\right)} \nabla \cdot\left(h_{1} \nabla \psi_{1}\right) .
$$

Then, noting that $Q_{1}=\tilde{Q}_{1}+\tilde{\tilde{Q}}_{1}$ with

$$
\begin{aligned}
& \tilde{Q}_{1}=\left(\begin{array}{cc}
\zeta I_{d \times d} & -\sqrt{\mu_{2}}(z+1) \nabla \zeta \\
-\sqrt{\mu_{2}}(z+1) \nabla \zeta^{T} & -\zeta
\end{array}\right), \\
& \tilde{\tilde{Q}}_{1}=\left(\begin{array}{cc}
-b I_{d \times d} & \sqrt{\mu_{2}} z \nabla b \\
\sqrt{\mu_{2}} z \nabla b^{T} & b
\end{array}\right),
\end{aligned}
$$

and because

$$
\begin{aligned}
-\nabla_{X, z}^{\mu_{2}} \cdot \tilde{Q}_{1} \nabla_{X, z}^{\mu_{2}} \Phi^{(0)} & =\Delta_{X, z}^{\mu_{2}}\left[(z+1) \zeta \partial_{z} \Phi^{(0)}\right], \\
-\left.\mathbf{e}_{z} \cdot \tilde{Q}_{1} \nabla_{X, z}^{\mu_{2}} \Phi^{(0)}\right|_{z=0} & =\left.\left[\mu_{2} \nabla \cdot\left(\zeta \nabla \Phi^{(0)}\right)+\partial_{z}\left((z+1) \zeta \partial_{z} \Phi^{(0)}\right)\right]\right|_{z=0}, \\
-\left.\mathbf{e}_{z} \cdot \tilde{Q}_{1} \nabla_{X, z}^{\mu_{2}} \Phi^{(0)}\right|_{z=-1} & =0,
\end{aligned}
$$

it follows that $\Phi^{(1)}=(z+1) \zeta \partial_{z} \Phi^{(0)}+u+v$, where $u$ solves the boundary value problem

$$
\left\{\begin{array}{l}
\Delta_{X, z}^{\mu_{2}} u=0, \\
\left.\partial_{z} u\right|_{z=0}=\frac{\mu^{2}}{3 \delta \varepsilon_{2}} \nabla \cdot\left(\Delta \nabla \psi_{1}\right)+\mu_{2} \nabla \cdot\left(\zeta \nabla \Phi^{(0)}\right),\left.\quad \partial_{z} u\right|_{z=-1}=0,
\end{array}\right.
$$

and $v$ solves the boundary value problem

$$
\left\{\begin{array}{l}
\Delta_{X, z}^{\mu_{2}} v=-\nabla_{X, z}^{\mu_{2}} \cdot \tilde{\tilde{Q}}_{1} \nabla_{X, z}^{\mu_{2}} \Phi^{(0)}, \\
\left.\partial_{z} v\right|_{z=0}=-\left.\mathbf{e}_{z} \cdot \tilde{Q}_{1} \nabla_{X, z}^{\mu_{2}} \Phi^{(0)}\right|_{z=0},\left.\quad \partial_{z} v\right|_{z=-1}=-\left.\mathbf{e}_{z} \cdot \tilde{Q}_{1} \nabla_{X, z}^{\mu_{2}} \Phi^{(0)}\right|_{z=-1} .
\end{array}\right.
$$

The problem for $u$ is obviously solved by

$$
\begin{aligned}
u(X, z)= & \frac{\cosh \left(\sqrt{\mu_{2}}(z+1)|D|\right)}{\cosh \left(\sqrt{\mu_{2}}|D|\right)} \frac{1}{|D| \tanh \left(\sqrt{\mu_{2}}|D|\right)} \\
& \times\left[\frac{\mu^{2}}{3 \delta \varepsilon_{2} \sqrt{\mu_{2}}} \nabla \cdot\left(\Delta \nabla \psi_{1}\right)+\sqrt{\mu_{2}} \nabla \cdot\left(\zeta \nabla \Phi^{(0)}\right)\right] .
\end{aligned}
$$


We now solve the boundary value problem for $v$. Since

$$
-\nabla_{X, z}^{\mu_{2}} \cdot \tilde{\tilde{Q}}_{1} \nabla_{X, z}^{\mu_{2}} \Phi^{(0)}=\Delta_{X, z}^{\mu_{2}}\left(-b z \partial_{z} \Phi^{(0)}\right)
$$

and

$$
-\mathbf{e}_{z} \cdot \tilde{\tilde{Q}}_{1} \nabla_{X, z}^{\mu_{2}} \Phi^{(0)}+\partial_{z}\left(b \partial_{z} \Phi^{(0)}\right)=-\mu_{2} z \nabla b \cdot \nabla \Phi^{(0)}+z b \partial_{z}^{2} \Phi^{(0)},
$$

we deduce that $v=v_{1}-b \partial_{z} \Phi^{(0)}$, where $v_{1}$ solves

$$
\left\{\begin{array}{l}
\Delta_{X, z}^{\mu_{2}} v_{1}=0, \\
\left.\partial_{z} v_{1}\right|_{z=0}=0, \\
\left.\partial_{z} v_{1}\right|_{z=-1}=\frac{\mu_{2} \sqrt{\mu}|D|}{\sinh \left(\sqrt{\mu_{2}}|D|\right)} b \nabla \cdot\left(h_{1} \nabla \psi_{1}\right),
\end{array}\right.
$$

which can be easily solved to obtain

$$
\begin{aligned}
v_{1}(X, z)= & \frac{\sqrt{\mu \mu_{2}}}{\sinh \left(\sqrt{\mu_{2}}|D|\right)} \frac{\cosh \left(\sqrt{\mu_{2}}|D| z\right)}{\cosh \left(\sqrt{\mu_{2}}|D|\right)} \frac{1}{\tanh \left(\sqrt{\mu_{2}}|D|\right)} \\
& \times\left[\nabla b \cdot \Pi\left(h_{1} \nabla \psi_{1}\right)+b \nabla \cdot\left(h_{1} \nabla \psi_{1}\right)\right] .
\end{aligned}
$$

Noting that in the Boussinesq/Boussinesq regime $\mu \sim \varepsilon \sim \mu_{2} \sim \varepsilon_{2} \ll 1$, we have

$$
\begin{aligned}
& \frac{1}{\tanh \left(\sqrt{\mu_{2}}|D|\right)} \sim \frac{1}{\sqrt{\mu_{2}}|D|} \frac{1}{1-\frac{1}{3} \mu_{2}|D|^{2}} \sim \frac{1+\frac{1}{3} \mu_{2}|D|^{2}}{\sqrt{\mu_{2}}|D|} \\
& \frac{1}{\sinh \left(2 \sqrt{\mu_{2}}|D|\right)} \sim \frac{1}{2 \sqrt{\mu_{2}}|D|} \frac{1}{1+\frac{2}{3} \mu_{2}|D|^{2}} \sim \frac{1-\frac{2}{3} \mu_{2}|D|^{2}}{2 \sqrt{\mu_{2}}|D|} .
\end{aligned}
$$

Substituting those into the expression for $\nabla\left(\left.\underline{\Phi}_{\text {app }}\right|_{z=0}\right)$,

$$
\nabla\left(\left.\underline{\Phi}_{\text {app }}\right|_{z=0}\right)=\left.\left[\nabla \Phi^{(0)}+(z+1) \nabla\left(\zeta \partial_{z} \Phi^{(0)}\right)+\nabla u+\nabla v_{1}-\nabla\left(b \partial_{z} \Phi^{(0)}\right)\right]\right|_{z=0},
$$

we obtain

$$
\begin{aligned}
\nabla\left(\left.\underline{\Phi}_{\mathrm{app}}\right|_{z=0}\right)= & -\delta \nabla \psi_{1}-\frac{1}{3} \mu \delta\left(1-\frac{1}{\delta^{2}}\right) \Delta \nabla \psi_{1}+\varepsilon_{2}(1+\delta) \Pi\left(\zeta \nabla \psi_{1}\right) \\
& +\varepsilon_{2} \delta \frac{1}{|D|^{2}}\left(\nabla\left(\nabla b . \nabla \psi_{1}\right)+\nabla\left(b \Delta \psi_{1}\right)\right)+O\left(\varepsilon^{2}\right) .
\end{aligned}
$$

We thus have the following

Proposition 2.2. Let $t_{0}>d / 2, s \geq t_{0}+1 / 2$, and $\zeta, b \in H^{s+3 / 2}\left(\mathbb{R}^{d}\right)$ be such that (2.10) and (2.11) are satisfied. Then, for all $\psi_{1}$ such that $\nabla \psi_{1} \in$ $H^{s+5 / 2}\left(\mathbb{R}^{d}\right)$, 


$$
\begin{aligned}
\mid \mathbf{H}^{\mu, \delta}[\varepsilon \zeta, \beta b] \psi_{1}-\left[-\delta \nabla \psi_{1}\right. & -\frac{1}{3} \mu \delta\left(1-\frac{1}{\delta^{2}}\right) \Delta \nabla \psi_{1}+\varepsilon_{2}(1+\delta) \Pi\left(\zeta \nabla \psi_{1}\right) \\
& \left.+\varepsilon_{2} \delta \frac{1}{|D|^{2}}\left(\nabla\left(\nabla b . \nabla \psi_{1}\right)+\nabla\left(b \Delta \psi_{1}\right)\right)\right]\left.\right|_{H^{s}} \\
\leq & \frac{\mu^{5 / 2}+\varepsilon_{2}^{2} \sqrt{\mu}}{\sqrt{\mu_{2}}} C\left(\frac{1}{H_{1}}, \frac{1}{H_{2}}, \delta^{\max }, \mu_{2}^{\max },|\zeta|_{H^{s+3 / 2}},|b|_{H^{s+3 / 2}}\right)\left|\nabla \psi_{1}\right|_{H^{s+5 / 2}} .
\end{aligned}
$$

This estimate is uniform with respect to $\varepsilon \in[0,1], \mu \in(0,1)$ and $\delta \in$ $\left(0, \delta^{\max }\right)$ such that $\mu_{2}=\mu / \delta^{2} \in\left(0, \mu_{2}^{\max }\right)$.

Proof. The proof is similar to the proof of Corollary 1 in [12], so it is omitted here. Note that $\sqrt{\mu}$ appears in the term $\varepsilon_{2}^{2} \sqrt{\mu}$ because of the expression for $\Phi^{(0)}$. This enables us to improve the precision of the expression for the operator $\mathbf{H}^{\mu, \delta}[\varepsilon \zeta, \beta b]$.

REMARK 2.1. It is interesting that if $b=0$ we get the same expression for $\mathbf{H}^{\mu, \delta}[\varepsilon \zeta]$ as in the case of a flat bottom [12], but with a higher precision $\left(O\left(\varepsilon^{2}\right)\right.$ instead of $O\left(\varepsilon^{3 / 2}\right)$ as in $\left.[12]\right)$.

2.3. Derivation of the Boussinesq/Boussinesq systems. In this subsection we give the asymptotic form of the equation (2.14) in the Boussinesq/Boussinesq regime. The asymptotic model is derived from (2.14) by replacing the operators $\mathbf{G}^{\mu}[\varepsilon \zeta], \mathbf{H}^{\mu, \delta}[\varepsilon \zeta, \beta b]$ and the term $\left(\sigma / \varrho_{2}\right) \nabla K(\zeta)$ by their asymptotic expansions and using a suitable "BBM trick" and a change of variable. First, we prove the following lemma which gives the asymptotic expansion of the term $\left(\sigma / \varrho_{2}\right) \nabla K(\zeta)$ containing the surface tension. Noting that we have $\varepsilon \sqrt{\mu} \ll 1$ in the present regime.

LEMMA 2.1. Let $\zeta \in H^{s+3}\left(\mathbb{R}^{d}\right)$ with $s>d / 2$. Then

$$
\left|\frac{\sigma}{\varrho_{2}} \nabla K(\zeta)-\varepsilon \sqrt{\mu} \nu \Delta \nabla \zeta\right|_{H^{s}} \leq(\varepsilon \sqrt{\mu})^{3} C\left(\mu^{\max }, \nu^{\max },|\zeta|_{H^{s+3}}\right) .
$$

This estimate is uniform with respect to $\varepsilon \in[0,1], \mu \in\left(0, \mu^{\max }\right)$ and $\nu \in$ $\left[0, \nu^{\max }\right]$.

Proof. If $d=1$, by a simple calculation and noticing that we are working with dimensionless variables and dimensionless unknowns, we get

$$
\frac{\sigma}{\varrho_{2}} \nabla K(\zeta)=\frac{a}{\lambda} \frac{\sigma}{\varrho_{2} \lambda^{2}} \frac{\zeta_{x x x}}{\left(1+\frac{a^{2}}{\lambda^{2}} \zeta_{x}^{2}\right)^{3 / 2}}-3 \frac{a^{3}}{\lambda^{3}} \frac{\sigma}{\varrho_{2} \lambda^{2}} \frac{\zeta_{x} \zeta_{x x}^{2}}{\left(1+\frac{a^{2}}{\lambda^{2}} \zeta_{x}^{2}\right)^{5 / 2}} .
$$

Noting that

$$
\frac{a^{2}}{\lambda^{2}}=\frac{a^{2}}{d_{1}^{2}} \frac{d_{1}^{2}}{\lambda^{2}}=\varepsilon^{2} \mu, \quad \nu=\frac{\sigma}{\varrho_{2} \lambda^{2}}
$$

and $H^{s}\left(\mathbb{R}^{d}\right)$ is an algebra for $s>d / 2$, using the Taylor formula we obtain the conclusion in the case $d=1$. The case $d=2$ is proved similarly. 
We now show that in the present regime, the internal waves equations (2.14) are consistent with the three-parameter family of Boussinesq/Boussinesq systems

$$
\left\{\begin{array}{c}
\left(1-\mu a_{2} \Delta\right) \partial_{t} \zeta+\frac{1}{\gamma+\delta} \nabla \cdot \mathbf{v}_{\alpha}+\varepsilon \frac{\delta^{2}-\gamma}{(\gamma+\delta)^{2}} \nabla \cdot\left(\zeta \mathbf{v}_{\alpha}\right) \\
\quad-\frac{\varepsilon \delta^{2}}{(\gamma+\delta)^{2}} \nabla \cdot\left(b \mathbf{v}_{\alpha}\right)+\mu a_{1} \nabla \cdot \Delta \mathbf{v}_{\alpha}=0 \\
\left(1-\mu a_{4} \Delta\right) \partial_{t} \mathbf{v}_{\alpha}+(1-\gamma) \nabla \zeta+\frac{\varepsilon}{2} \frac{\delta^{2}-\gamma}{(\gamma+\delta)^{2}} \nabla\left|\mathbf{v}_{\alpha}\right|^{2} \\
+\mu a_{3}(1-\gamma) \Delta \nabla \zeta-\varepsilon \sqrt{\mu} \nu \Delta \nabla \zeta=0,
\end{array}\right.
$$

where $\mathbf{v}_{\alpha}=(1-\mu \alpha \Delta)^{-1} \mathbf{v}$ and the constants $a_{1}, a_{2}, a_{3}$ and $a_{4}$ are defined below.

Theorem 2.1. Let $0<c^{\min }<c^{\max }, 0<\mu_{2}^{\min }<\mu_{2}^{\max }$, and set

$$
\begin{aligned}
& a_{1}=\frac{\left(1-\alpha_{1}\right)(1+\gamma \delta)-3 \delta \alpha(\gamma+\delta)}{3 \delta(\gamma+\delta)^{2}}, \quad a_{2}=\frac{\gamma \alpha_{1}}{3(\gamma+\delta)}, \\
& a_{3}=\alpha \alpha_{2}, \quad a_{4}=\alpha\left(1-\alpha_{2}\right),
\end{aligned}
$$

with $\alpha_{1}, \alpha \geq 0$ and $\alpha_{2} \leq 1$. With these choices of parameters, the internal waves equations (2.14) are consistent with the Boussinesq/Boussinesq equations (2.17) in the sense of Definition 3, with $O\left(\varepsilon^{2}\right)$ precision, and uniformly with respect to $\varepsilon \in[0,1], \mu, \delta \in(0,1)$ satisfying the conditions

$$
c^{\min } \leq \frac{\varepsilon}{\mu} \leq c^{\max }, \quad \mu_{2}^{\min } \leq \frac{\mu}{\delta^{2}} \leq \mu_{2}^{\max },
$$

and $\nu \in\left[0, \nu^{\max }\right]$.

REMARK 2.2. One can give a more precise estimate of the precision, as in Proposition 2.2 for instance. It simplifies the exposition to use the notation $O\left(\varepsilon^{2}\right)$ and the associated rough estimate of the precision. We follow this policy throughout the discussion.

REMARK 2.3. When $b=0$ and $\nu=0$, we recover of course the Boussinesq/Boussinesq systems derived in [12] for internal waves in the case of a flat bottom and absence of surface tension. It is interesting that the precision of (2.17) is higher than the corresponding one in [12] $\left(O\left(\varepsilon^{2}\right)\right.$ instead of $\left.O\left(\varepsilon^{3 / 2}\right)\right)$.

Remark 2.4. Taking $\gamma=0, \delta=1$ and $\nu=0$ in the Boussinesq/Boussinesq equations (2.17), we reduce them to the system

$$
\left\{\begin{array}{l}
\left(1-\mu \frac{\alpha_{1}}{3} \Delta\right) \partial_{t} \zeta+\nabla \cdot \mathbf{v}+\varepsilon \nabla \cdot((\zeta-b) \mathbf{v})+\mu \frac{1-\alpha_{1}-3 \alpha}{3} \Delta \nabla \cdot \mathbf{v}=0, \\
\left(1-\mu \alpha\left(1-\alpha_{2}\right) \Delta\right) \partial_{t} \mathbf{v}+\nabla \zeta+\frac{\varepsilon}{2} \nabla|\mathbf{v}|^{2}+\mu \alpha \alpha_{2} \Delta \nabla \zeta=0,
\end{array}\right.
$$


which is exactly the family of formally equivalent Boussinesq systems derived in [15] for the surface waves in the case of nonflat bottoms and absence of surface tension. In particular, when $b=0$ we recover the Boussinesq systems (1.1) upon noting that we have taken the Stokes number $S=a \lambda^{2} / d_{1}^{3}=1$, i.e. $\varepsilon=\mu$, when deriving (1.1).

REMARK 2.5. The dispersion relation associated to (2.17) is

$$
\omega^{2}=|\mathbf{k}|^{2} \frac{\left(\frac{1}{\gamma+\delta}-\mu a_{1}|\mathbf{k}|^{2}\right)\left[(1-\gamma)\left(1-\mu a_{3}|\mathbf{k}|^{2}\right)+\varepsilon \sqrt{\mu} \nu|\mathbf{k}|^{2}\right]}{\left(1+\mu a_{2}|\mathbf{k}|^{2}\right)\left(1+\mu a_{4}|\mathbf{k}|^{2}\right)} .
$$

It follows that (2.17) is linearly well-posed when $a_{1} \leq 0, a_{3} \leq \varepsilon \nu /((1-\gamma) \sqrt{\mu})$ and $a_{2}, a_{4} \geq 0$. Recall that in the absence of surface tension, the Boussinesq/Boussinesq system is linearly well-posed when $a_{1}, a_{3} \leq 0$ and $a_{2}, a_{4} \geq 0$ (see [12]). Thus, the surface tension makes the well-posedness results better.

Proof. The proof is in several steps, corresponding to particular assumptions about the parameters $\alpha_{1}, \alpha_{2}$ and $\alpha$. In this regime, we have $\varepsilon \sim \mu \sim \varepsilon_{2} \sim \mu_{2}$ as $\varepsilon \rightarrow 0$.

SteP 1: $\alpha_{1}=\alpha=\alpha_{2}=0$. From the expansion of the DirichletNeumann operator and Lemma 2.1, it follows that

$$
\left\{\begin{aligned}
\partial_{t} \zeta & -\nabla \cdot\left((1-\varepsilon \zeta) \nabla \psi_{1}\right)-\frac{\mu}{3} \nabla \cdot\left(\Delta \nabla \psi_{1}\right)=O\left(\varepsilon^{2}\right), \\
\partial_{t} \mathbf{v} & +(1-\gamma) \nabla \zeta \\
& +\frac{\varepsilon}{2} \nabla\left(\left|\mathbf{H}^{\mu, \delta}[\varepsilon \zeta, \beta b] \psi_{1}\right|^{2}-\gamma\left|\nabla \psi_{1}\right|^{2}\right)-\varepsilon \sqrt{\mu} \nu \Delta \nabla \zeta=O\left(\varepsilon^{2}\right),
\end{aligned}\right.
$$

where the fact that $O(\mu)=O(\varepsilon)$ has been used. From the relation $\mathbf{H}^{\mu, \delta}[\varepsilon \zeta, \beta b] \psi_{1}=\mathbf{v}+\gamma \nabla \psi_{1}$ and Proposition 2.2, it is seen that

$$
\begin{aligned}
\nabla \psi_{1}= & -\frac{1}{\gamma+\delta}\left[1+\mu \frac{1}{3 \delta} \frac{1-\delta^{2}}{(\gamma+\delta)} \Delta+\varepsilon_{2} \frac{1+\delta}{\gamma+\delta} \Pi[\zeta .]\right] \mathbf{v} \\
& -\frac{\varepsilon_{2} \delta}{(\gamma+\delta)^{2}}\left[\frac{\nabla}{|D|^{2}}(\nabla b . \mathbf{v})+\frac{\nabla}{|D|^{2}}(b \nabla . \mathbf{v})\right]+O\left(\varepsilon^{2}\right) .
\end{aligned}
$$

Substituting this into the above system yields the result.

STEP 2: $\alpha_{1} \geq 0, \alpha=\alpha_{2}=0$. Here we use the classical BBM trick. It is clear from the first equation that

$$
\nabla \cdot \mathbf{v}=\left(1-\alpha_{1}\right) \nabla \cdot \mathbf{v}-\alpha_{1}(\gamma+\delta) \partial_{t} \zeta+O(\varepsilon) .
$$

Replacing $\nabla \cdot \mathbf{v}$ by this expression in the third-derivative term of the first equation of the system derived in Step 1 leads to the desired result.

STEP 3: $\alpha_{1}, \alpha \geq 0, \alpha_{2}=0$. Replacing $\mathbf{v}$ by $(1-\mu \alpha \Delta) \mathbf{v}_{\alpha}$ in the system derived in Step 2, and neglecting the $O\left(\varepsilon^{2}\right)$ terms, is all that is required in this case. 
SteP 4: $\alpha_{1}, \alpha \geq 0, \alpha_{2} \leq 1$. We again use the BBM trick. From the second equation in the system derived in Step 3, one finds that for all $\alpha_{2} \leq 1$,

$$
\partial_{t} \mathbf{v}_{\alpha}=\left(1-\alpha_{2}\right) \partial_{t} \mathbf{v}_{\alpha}-\alpha_{2}(1-\gamma) \nabla \zeta+O(\varepsilon) .
$$

If this is substituted into the system derived in Step 3, the result follows.

3. Well-posedness of the Boussinesq/Boussinesq systems. For clarity, we will write $V$ instead of $\mathbf{v}_{\alpha}:=(1-\mu \alpha \Delta)^{-1} \mathbf{v}$. Then the system (2.17) has the following form:

$$
\left\{\begin{array}{c}
\left(1-\mu a_{2} \Delta\right) \partial_{t} \zeta+\frac{1}{\gamma+\delta} \nabla \cdot V+\varepsilon \frac{\delta^{2}-\gamma}{(\gamma+\delta)^{2}} \nabla \cdot(\zeta V) \\
-\frac{\varepsilon \delta^{2}}{(\gamma+\delta)^{2}} \nabla \cdot(b V)+\mu a_{1} \Delta \nabla \cdot V=0, \\
\left(1-\mu a_{4} \Delta\right) \partial_{t} V+(1-\gamma) \nabla \zeta+\frac{\varepsilon}{2} \frac{\delta^{2}-\gamma}{(\gamma+\delta)^{2}} \nabla|V|^{2} \\
+\mu a_{3}(1-\gamma) \Delta \nabla \zeta-\varepsilon \sqrt{\mu} \nu \Delta \nabla \zeta=0 .
\end{array}\right.
$$

The well-posedness results will be formulated for two unknowns $\zeta$ and $V$.

Recall (see Remark 2.5) that the system (3.1) is linearly well-posed when

$$
a_{2}, a_{4} \geq 0, \quad a_{1} \leq 0, \quad a_{3} \leq \frac{\varepsilon \nu}{(1-\gamma) \sqrt{\mu}} .
$$

It is reasonable to argue that linear well-posedness is a natural necessary requirement for nonlinear well-posedness. In this section, following the general lines of the analogous proof of [9], we will show that the systems (3.1) which are linearly well-posed are in fact locally nonlinearly well-posed in suitable Sobolev classes.

3.1. The weakly dispersive Boussinesq/Boussinesq systems. We call the Boussinesq/Boussinesq systems (3.1) weakly dispersive when

$$
a_{2}>0 \text { and } a_{4}>0 .
$$

First, we consider the cases where

$$
a_{2}, a_{4}>0, \quad a_{1}=0, \quad a_{3}=\frac{\varepsilon \nu}{(1-\gamma) \sqrt{\mu}} .
$$

In these cases the system (3.1) can be written in the form

$$
\left\{\begin{aligned}
& \partial_{t} \zeta+\left(I-\mu a_{2} \Delta\right)^{-1}\left[\frac{1}{\gamma+\delta} \nabla \cdot V+\right. \varepsilon \frac{\delta^{2}-\gamma}{(\gamma+\delta)^{2}} \nabla \cdot(\zeta V) \\
&-\left.\frac{\varepsilon \delta^{2}}{(\gamma+\delta)^{2}} \nabla \cdot(b V)\right]=0 \\
& \partial_{t} V+\left(I-\mu a_{4} \Delta\right)^{-1}\left[(1-\gamma) \nabla \zeta+\frac{\varepsilon}{2} \frac{\delta^{2}-\gamma}{(\gamma+\delta)^{2}} \nabla|V|^{2}\right]=0
\end{aligned}\right.
$$

Using a contraction-mapping argument, we prove the following theorem. 
Theorem 3.1. Let $s>0$ and $b \in H^{s}\left(\mathbb{R}^{2}\right),\left(\zeta_{0}, V_{0}\right) \in H^{s}\left(\mathbb{R}^{2}\right)^{3}$. Then there exist $T>0$ and a unique solution

$$
(\zeta, V) \in X_{T}=C\left([0, T] ; H^{s}\left(\mathbb{R}^{2}\right)\right)^{3}
$$

of (3.2) with initial condition $\left(\zeta_{0}, V_{0}\right)$.

Proof. Let $(\tilde{\zeta}, \tilde{V}) \in X_{T}$ define the operator $\mathcal{F}$ by

$$
\begin{aligned}
& \left(\begin{array}{c}
\zeta \\
V
\end{array}\right)=\mathcal{F}(\tilde{\zeta}, \tilde{V})= \\
& \left(\begin{array}{c}
\zeta_{0} \\
V_{0}
\end{array}\right)-\left(\begin{array}{c}
\int_{0}^{t}\left(I-\mu a_{2} \Delta\right)^{-1} \nabla \cdot\left[\frac{1}{\gamma+\delta} V+\varepsilon \frac{\delta^{2}-\gamma}{(\gamma+\delta)^{2}}(\tilde{\zeta} \tilde{V})-\frac{\varepsilon \delta^{2}}{(\gamma+\delta)^{2}}(b \tilde{V})\right](X, s) d s \\
\int_{0}^{t}\left[\left(I-\mu a_{4} \Delta\right)^{-1} \nabla\left[(1-\gamma) \zeta+\frac{\varepsilon}{2} \frac{\delta^{2}-\gamma}{(\gamma+\delta)^{2}}|V|^{2}\right]\right](X, s) d s
\end{array}\right) .
\end{aligned}
$$

In order to estimate the integrands appearing in the definition of $\mathcal{F}$, we will use the following lemma, proved by Grisvard [22].

Lemma 3.1. Let $s_{1}, s_{2}, s_{3} \in \mathbb{R}$ be such that $s_{1}, s_{2} \geq s_{3}, s_{1}+s_{2} \geq 0$, $s_{1}+s_{2}-s_{3}>d / 2$. Then $(f, g) \mapsto f g$ is bilinear continuous from $H^{s_{1}}\left(\mathbb{R}^{d}\right) \times$ $H^{s_{2}}\left(\mathbb{R}^{d}\right)$ into $H^{s_{3}}\left(\mathbb{R}^{d}\right)$.

This lemma implies that for $f, g \in H^{s}\left(\mathbb{R}^{2}\right), s>0$, we have

$$
\|f g\|_{s-1} \leq C\|f\|_{s}\|g\|_{s},
$$

where $\|\cdot\|_{s}$ denotes the norm of $H^{s}\left(\mathbb{R}^{2}\right)$.

Thus, there are constants $C_{1}, C_{2}$ depending only on $a_{2}, \mu$, and constants $C_{3}, C_{4}$ depending only on $a_{4}, \mu$ such that for $i=1,2$,

$$
\begin{aligned}
& \left\|\left(I-\mu a_{2} \Delta\right)^{-1} \partial_{i}(f g)\right\|_{s} \leq C_{1}\|f g\|_{s-1} \leq C_{2}\|f\|_{s}\|g\|_{s}, \\
& \left\|\left(I-\mu a_{4} \Delta\right)^{-1} \partial_{i}(f g)\right\|_{s} \leq C_{3}\|f g\|_{s-1} \leq C_{4}\|f\|_{s}\|g\|_{s},
\end{aligned}
$$

where $\partial_{1}=\partial_{x}, \partial_{2}=\partial_{y}$. It thus follows that there is a constant $C$ depending only on $a_{2}, a_{4}, \mu, \gamma, \delta, \varepsilon$ and $\|b\|_{s}$ such that

$$
\begin{aligned}
\sup _{0 \leq t \leq T} & \left\|\mathcal{F}\left(\zeta_{1}, V_{1}\right)-\mathcal{F}\left(\zeta_{2}, V_{2}\right)\right\|_{s} \\
& \leq T C\left(1+\left\|\left(\zeta_{1}, V_{1}\right)\right\|_{X_{T}}+\left\|\left(\zeta_{2}, V_{2}\right)\right\|_{X_{T}}\right)\left\|\left(\zeta_{1}, V_{1}\right)-\left(\zeta_{2}, V_{2}\right)\right\|_{X_{T}} \\
& \leq T C(1+2 R)\left\|\left(\zeta_{1}, V_{1}\right)-\left(\zeta_{2}, V_{2}\right)\right\|_{X_{T}}
\end{aligned}
$$

provided that $\left(\zeta_{1}, V_{1}\right)$ and $\left(\zeta_{2}, V_{2}\right)$ are in the closed ball $\bar{B}_{R}$ of radius $R$ centred at 0 in $X_{T}$. Choosing $R=2\left\|\left(\zeta_{0}, V_{0}\right)\right\|_{X_{T}}$ and $T=1 /(2 C(1+2 R))$, it is clear that

$$
\left\|\mathcal{F}\left(\zeta_{1}, V_{1}\right)-\mathcal{F}\left(\zeta_{2}, V_{2}\right)\right\|_{X_{T}} \leq \frac{1}{2}\left\|\left(\zeta_{1}, V_{1}\right)-\left(\zeta_{2}, V_{2}\right)\right\|_{X_{T}}
$$


and

$$
\begin{aligned}
\left\|\mathcal{F}\left(\zeta_{1}, V_{1}\right)\right\|_{X_{T}} & =\left\|\mathcal{F}\left(\zeta_{1}, V_{1}\right)-\mathcal{F}(0,0)+\left(\zeta_{0}, V_{0}\right)\right\|_{X_{T}} \\
& \leq T C(1+2 R) R+\left\|\left(\zeta_{0}, V_{0}\right)\right\|_{X_{T}} \leq R .
\end{aligned}
$$

Therefore $\mathcal{F}$ is a contraction maping from $\bar{B}_{R}$ to $\bar{B}_{R}$ in $X_{T}$, and the theorem follows.

We now turn to other cases wherein $a_{2}, a_{4}>0$. Realizing (3.1) as systems coupled only through nonlinear terms and using a contraction-mapping argument, we will prove the following theorem.

TheOREM 3.2. Let $s>0$ and $b \in H^{s}\left(\mathbb{R}^{2}\right)$.

(i) Assume $a_{2}, a_{4}>0, a_{1}<0, a_{3}<\varepsilon \nu /((1-\gamma) \sqrt{\mu})$. Let $\left(\zeta_{0}, V_{0}\right) \in$ $H^{s}\left(\mathbb{R}^{2}\right)^{3}$. Then there exist $T>0$ and a unique solution

$$
(\zeta, V) \in X_{T}=C\left([0, T] ; H^{s}\left(\mathbb{R}^{2}\right)\right)^{3}
$$

of (3.1) with initial condition $\left(\zeta_{0}, V_{0}\right)$.

(ii) Assume $a_{2}, a_{4}>0, a_{1}=0, a_{3}<\varepsilon \nu /((1-\gamma) \sqrt{\mu})$. Let $\left(\zeta_{0}, V_{0}\right) \in$ $H^{s+1}\left(\mathbb{R}^{2}\right) \times H^{s}\left(\mathbb{R}^{2}\right)^{2}$. Then there exist $T>0$ and a unique solution

$$
(\zeta, V) \in X_{T}=C\left([0, T] ; H^{s+1}\left(\mathbb{R}^{2}\right)\right) \times C\left([0, T] ; H^{s}\left(\mathbb{R}^{2}\right)\right)^{2}
$$

of (3.1) with initial condition $\left(\zeta_{0}, V_{0}\right)$.

(iii) Assume $a_{2}, a_{4}>0, a_{1}<0, a_{3}=\varepsilon \nu /((1-\gamma) \sqrt{\mu})$. Let $\left(\zeta_{0}, V_{0}\right) \in$ $H^{s-1}\left(\mathbb{R}^{2}\right) \times H^{s}\left(\mathbb{R}^{2}\right)^{2}$. Then there exist $T>0$ and a unique solution

$$
(\zeta, V) \in X_{T}=C\left([0, T] ; H^{s-1}\left(\mathbb{R}^{2}\right)\right) \times C\left([0, T] ; H^{s}\left(\mathbb{R}^{2}\right)\right)^{2}
$$

of (3.1) with initial condition $\left(\zeta_{0}, V_{0}\right)$.

Proof. Taking the Fourier transform with respect to $X$, we can write (3.1) in the form

$$
\begin{aligned}
\frac{d}{d t}\left(\begin{array}{c}
\hat{\zeta} \\
\hat{v}_{1} \\
\hat{v}_{2}
\end{array}\right)+i|\mathbf{k}| \mathcal{A}(\mathbf{k})\left(\begin{array}{c}
\hat{\zeta} \\
\hat{v}_{1} \\
\hat{v}_{2}
\end{array}\right) \\
+i\left(\begin{array}{c}
\frac{\varepsilon \frac{\delta^{2}-\gamma}{(\gamma+\delta)^{2}}\left(k_{1} \widehat{\zeta v_{1}}+k_{2} \widehat{\left.\zeta v_{2}\right)}-\frac{\varepsilon \delta^{2}}{(\gamma+\delta)^{2}}\left(k_{1} \widehat{b v_{1}}+k_{2} \widehat{b v_{2}}\right)\right.}{1+\mu a_{2}|\mathbf{k}|^{2}} \\
\frac{\frac{\varepsilon}{2} \frac{\delta^{2}-\gamma}{(\gamma+\delta)^{2}}}{1+\mu a_{4}|\mathbf{k}|^{2}} k_{1} \mid \widehat{\left.V\right|^{2}} \\
\frac{\varepsilon}{\frac{\varepsilon}{2} \frac{\delta^{2}-\gamma}{(\gamma+\delta)^{2}}} \\
1+\mu a_{4}|\mathbf{k}|^{2} \\
1+\widehat{\left.V\right|^{2}}
\end{array}\right)=0,
\end{aligned}
$$


where $V=\left(v_{1}, v_{2}\right), \mathbf{k}=\left(k_{1}, k_{2}\right)$, and

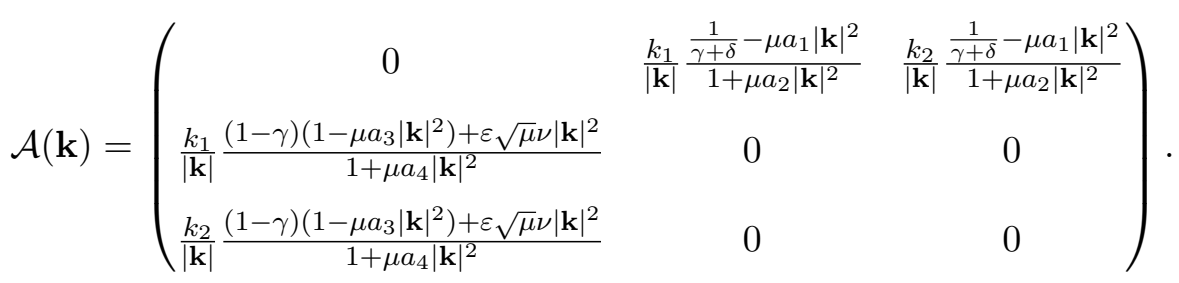

The eigenvalues of $\mathcal{A}(\mathbf{k})$ are $\{0, \pm \sigma(\mathbf{k})\}$, where

$$
\sigma(\mathbf{k})=\left[\frac{\left(\frac{1}{\gamma+\delta}-\mu a_{1}|\mathbf{k}|^{2}\right)\left[(1-\gamma)\left(1-\mu a_{3}|\mathbf{k}|^{2}\right)+\varepsilon \sqrt{\mu} \nu|\mathbf{k}|^{2}\right]}{\left(1+\mu a_{2}|\mathbf{k}|^{2}\right)\left(1+\mu a_{4}|\mathbf{k}|^{2}\right)}\right]^{1 / 2} .
$$

Diagonalize the above system:

$$
P^{-1}(\mathbf{k}) \mathcal{A}(\mathbf{k}) P(\mathbf{k})=\left(\begin{array}{ccc}
0 & 0 & 0 \\
0 & \sigma(\mathbf{k}) & 0 \\
0 & 0 & -\sigma(\mathbf{k})
\end{array}\right),
$$

with

$$
\begin{aligned}
P(\mathbf{k}) & =\left(\begin{array}{ccc}
0 & \alpha(\mathbf{k}) & -\alpha(\mathbf{k}) \\
-\frac{k_{2}}{|\mathbf{k}|} & \frac{k_{1}}{|\mathbf{k}|} & \frac{k_{1}}{|\mathbf{k}|} \\
\frac{k_{1}}{|\mathbf{k}|} & \frac{k_{2}}{|\mathbf{k}|} & \frac{k_{2}}{|\mathbf{k}|}
\end{array}\right), \\
P^{-1}(\mathbf{k}) & =\frac{1}{2 \alpha(\mathbf{k})}\left(\begin{array}{ccc}
0 & -2 \alpha(\mathbf{k}) \frac{k_{2}}{|\mathbf{k}|} & 2 \alpha(\mathbf{k}) \frac{k_{1}}{|\mathbf{k}|} \\
1 & \alpha(\mathbf{k}) \frac{k_{1}}{|\mathbf{k}|} & \alpha(\mathbf{k}) \frac{k_{2}}{|\mathbf{k}|} \\
-1 & \alpha(\mathbf{k}) \frac{k_{1}}{|\mathbf{k}|} & \alpha(\mathbf{k}) \frac{k_{2}}{|\mathbf{k}|}
\end{array}\right),
\end{aligned}
$$

where

$$
\alpha(\mathbf{k})=\left[\frac{\left(\frac{1}{\gamma+\delta}-\mu a_{1}|\mathbf{k}|^{2}\right)\left(1+\mu a_{4}|\mathbf{k}|^{2}\right)}{\left[(1-\gamma)\left(1-\mu a_{3}|\mathbf{k}|^{2}\right)+\varepsilon \sqrt{\mu} \nu|\mathbf{k}|^{2}\right]\left(1+\mu a_{2}|\mathbf{k}|^{2}\right)}\right]^{1 / 2} .
$$

Performing the change of variables

$$
\left(\begin{array}{c}
\hat{\mu} \\
\hat{\nu}_{1} \\
\hat{\nu}_{2}
\end{array}\right)=P^{-1}\left(\begin{array}{c}
\hat{\zeta} \\
\hat{v}_{1} \\
\hat{v}_{2}
\end{array}\right)
$$

we have 


$$
\begin{aligned}
& \frac{d}{d t}\left(\begin{array}{c}
\hat{\mu} \\
\hat{\nu}_{1} \\
\hat{\nu}_{2}
\end{array}\right)+i|\mathbf{k}|\left(\begin{array}{ccc}
0 & 0 & 0 \\
0 & \sigma(\mathbf{k}) & 0 \\
0 & 0 & -\sigma(\mathbf{k})
\end{array}\right)\left(\begin{array}{l}
\hat{\mu} \\
\hat{\nu_{1}} \\
\hat{\nu_{2}}
\end{array}\right) \\
& =-i P^{-1}(\mathbf{k})\left(\begin{array}{c}
\frac{\varepsilon \frac{\delta^{2}-\gamma}{(\gamma+\delta)^{2}}\left(k_{1} \widehat{\zeta v_{1}}+k_{2} \widehat{\zeta v_{2}}\right)-\frac{\delta^{2}}{(\gamma+\delta)^{2}}\left(k_{1} \widehat{b v_{1}}+k_{2} \widehat{b v_{2}}\right)}{1+\mu a_{2}|\mathbf{k}|^{2}} \\
\frac{\frac{\varepsilon}{2} \frac{\delta^{2}-\gamma}{(\gamma+\delta)^{2}}}{1+\mu a_{4}|\mathbf{k}|^{2}} k_{1} \mid \widehat{\left.V\right|^{2}} \\
\frac{\frac{\varepsilon}{2} \frac{\delta^{2}-\gamma}{(\gamma+\delta)^{2}}}{1+\mu a_{4}|\mathbf{k}|^{2}} k_{2} \mid \widehat{\left.V\right|^{2}}
\end{array}\right) .
\end{aligned}
$$

We get

$$
\begin{aligned}
\hat{\mu}(k) & =-\frac{k_{2}}{|\mathbf{k}|} \hat{v}_{1}(\mathbf{k})+\frac{k_{1}}{|\mathbf{k}|} \hat{v}_{2}(\mathbf{k}), \\
\hat{\nu}_{1}(\mathbf{k}) & =\frac{1}{2 \alpha(\mathbf{k})} \hat{\zeta}(\mathbf{k})+\frac{1}{2} \frac{k_{1}}{|\mathbf{k}|} \hat{v}_{1}(\mathbf{k})+\frac{1}{2} \frac{k_{2}}{|\mathbf{k}|} \hat{v}_{2}(\mathbf{k}), \\
\hat{\nu}_{2}(\mathbf{k}) & =-\frac{1}{2 \alpha(\mathbf{k})} \hat{\zeta}(\mathbf{k})+\frac{1}{2} \frac{k_{1}}{|\mathbf{k}|} \hat{v}_{1}(\mathbf{k})+\frac{1}{2} \frac{k_{2}}{|\mathbf{k}|} \hat{v}_{2}(\mathbf{k}) .
\end{aligned}
$$

Thus, with $H^{s}=H^{s}\left(\mathbb{R}^{2}\right)$, we have:

- If $\alpha(\mathbf{k})$ is of order 0, i.e. $a_{2}, a_{4}>0, a_{1}<0, a_{3}<\varepsilon \nu /((1-\gamma) \sqrt{\mu})$, then

$$
\left(\zeta, v_{1}, v_{2}\right) \in H^{s} \times H^{s} \times H^{s} \Leftrightarrow\left(\mu, \nu_{1}, \nu_{2}\right) \in H^{s} \times H^{s} \times H^{s} .
$$

- If $\alpha(\mathbf{k})$ is of order -1 , i.e. $a_{2}, a_{4}>0, a_{1}=0, a_{3}<\varepsilon \nu /((1-\gamma) \sqrt{\mu})$, then

$$
\left(\zeta, v_{1}, v_{2}\right) \in H^{s+1} \times H^{s} \times H^{s} \Leftrightarrow\left(\mu, \nu_{1}, \nu_{2}\right) \in H^{s} \times H^{s} \times H^{s} .
$$

- If $\alpha(\mathbf{k})$ is of order +1 , i.e. $a_{2}, a_{4}>0, a_{1}<0, a_{3}=\varepsilon \nu /((1-\gamma) \sqrt{\mu})$, then

$$
\left(\zeta, v_{1}, v_{2}\right) \in H^{s-1} \times H^{s} \times H^{s} \Leftrightarrow\left(\mu, \nu_{1}, \nu_{2}\right) \in H^{s} \times H^{s} \times H^{s} .
$$

Below we give the details in the case where $\alpha(\mathbf{k})$ is of order 0 . The other cases follow analogously.

Taking the inverse Fourier transform, we can write (3.6) in the form

$$
\frac{d}{d t}\left(\begin{array}{c}
\mu \\
\nu_{1} \\
\nu_{2}
\end{array}\right)+\mathcal{B}\left(\begin{array}{c}
\mu \\
\nu_{1} \\
\nu_{2}
\end{array}\right)=\mathcal{F}\left(\begin{array}{c}
\mu \\
\nu_{1} \\
\nu_{2}
\end{array}\right),
$$


where $\mathcal{B}$ is the skew-adjoint matrix operator with symbol

$$
i|\mathbf{k}|\left(\begin{array}{ccc}
0 & 0 & 0 \\
0 & \sigma(\mathbf{k}) & 0 \\
0 & 0 & -\sigma(\mathbf{k})
\end{array}\right)
$$

and $\mathcal{F}$ is the nonlinear term with Fourier transform given by the right-hand side of (3.6). In order to write an explicit expression of the nonlinear term $\mathcal{F}$, we note that in terms of $\left(\hat{\mu}, \hat{\nu}_{1}, \hat{\nu}_{2}\right)$,

$$
\left(\begin{array}{c}
\hat{\zeta} \\
\hat{v}_{1} \\
\hat{v}_{2}
\end{array}\right)=\left(\begin{array}{c}
\alpha(\mathbf{k}) \hat{\nu}_{1}(\mathbf{k})-\alpha(\mathbf{k}) \hat{\nu}_{2}(\mathbf{k}) \\
-\frac{k_{2}}{|\mathbf{k}|} \hat{\mu}(\mathbf{k})+\frac{k_{1}}{|\mathbf{k}|} \hat{\nu}_{1}(\mathbf{k})+\frac{k_{1}}{|\mathbf{k}|} \hat{\nu}_{2}(\mathbf{k}) \\
\frac{k_{1}}{|\mathbf{k}|} \hat{\mu}(\mathbf{k})+\frac{k_{2}}{|\mathbf{k}|} \hat{\nu}_{1}(\mathbf{k})+\frac{k_{2}}{|\mathbf{k}|} \hat{\nu}_{2}(\mathbf{k})
\end{array}\right) .
$$

Hence

$$
\left(\begin{array}{c}
\zeta \\
v_{1} \\
v_{2}
\end{array}\right)=\left(\begin{array}{c}
\alpha(D) \nu_{1}-\alpha(D) \nu_{2} \\
-R_{2} \mu+R_{1} \nu_{1}+R_{1} \nu_{2} \\
R_{1} \mu+R_{2} \nu_{1}+R_{2} \nu_{2}
\end{array}\right),
$$

where $\alpha(D)$ is the multiplier defined as follows:

$$
\widehat{\alpha(D) f}(\mathbf{k})=\alpha(\mathbf{k}) \hat{f}(\mathbf{k}),
$$

and $R_{1}, R_{2}$ are the Riesz transforms defined by

$$
\widehat{R_{1} f}(\mathbf{k})=\frac{k_{1}}{|\mathbf{k}|} \hat{f}(\mathbf{k}), \quad \widehat{R_{2} f}(\mathbf{k})=\frac{k_{2}}{|\mathbf{k}|} \hat{f}(\mathbf{k}) .
$$

It is clear that $\alpha(D)$ and $R_{i}(i=1,2)$ are bounded operators from $H^{s}$ to $H^{s}$ for all $s \in \mathbb{R}$. We now get the expression of $\mathcal{F}$ as follows:

$$
\begin{aligned}
& \mathcal{F}\left(\begin{array}{c}
\mu \\
\nu_{1} \\
\nu_{2}
\end{array}\right)= \\
& -\mathcal{P}^{-1}\left(\begin{array}{c}
\left(1-\mu a_{2} \Delta\right)^{-1}\left[\varepsilon \frac{\delta^{2}-\gamma}{(\gamma+\delta)^{2}}\left(\partial_{x}\left(\zeta v_{1}\right)+\partial_{y}\left(\zeta v_{2}\right)\right)-\frac{\delta^{2}}{(\gamma+\delta)^{2}}\left(\partial_{x}\left(b v_{1}\right)+\partial_{y}\left(b v_{2}\right)\right)\right] \\
\frac{\varepsilon}{2} \frac{\delta^{2}-\gamma}{(\gamma+\delta)^{2}}\left(1-\mu a_{4} \Delta\right)^{-1} \partial_{x}\left(v_{1}^{2}+v_{2}^{2}\right) \\
\frac{\varepsilon}{2} \frac{\delta^{2}-\gamma}{(\gamma+\delta)^{2}}\left(1-\mu a_{4} \Delta\right)^{-1} \partial_{y}\left(v_{1}^{2}+v_{2}^{2}\right)
\end{array}\right),
\end{aligned}
$$

where $\left(\zeta, v_{1}, v_{2}\right)$ are related to $\left(\mu, \nu_{1}, \nu_{2}\right)$ by $(3.8)$, and $\mathcal{P}^{-1}$ is the pseudo-differential operator with symbol $P^{-1}$. If $\alpha(\mathbf{k})$ is of order 0 , all the pseudo-differential operators involved are of order 0 . So is $P^{-1}(\mathbf{k})$. Using (3.4), (3.5) and the boundedness of $\mathcal{P}^{-1}, \alpha(D), R_{1}, R_{2}$, one can see that $\mathcal{F}$ is bilinear continuous from $H^{s} \times H^{s} \times H^{s}$ into itself for $s>0$. Denoting by $S(t)$ the 
semigroup generated by $\mathcal{B}$, it is clear that $S(t)$ is a unitary group on $H^{s}\left(\mathbb{R}^{2}\right)^{3}$ for all $s \in \mathbb{R}$. Set

$$
U=\left(\begin{array}{c}
\mu \\
\nu_{1} \\
\nu_{2}
\end{array}\right) .
$$

Let $U_{0}$ be related to the initial data $\left(\zeta_{0}, V_{0}\right)$ by (3.8). By Duhamel's formula, (3.7) completed with initial value $U_{0}$ is equivalent to

$$
U(t)=S(t) U_{0}+\int_{0}^{t} S(t-\tau) \mathcal{F}(U) d \tau .
$$

A function $U \in C\left([0, T] ; H^{s}\left(\mathbb{R}^{2}\right)^{3}\right)$ satisfying this integral equation is called an integral solution (or a mild solution) of (3.7). Since $\mathcal{F}$ is locally Lipschitz continuous (see below), by a result in [31, p. 190], it is known that if $U_{0} \in$ $D(\mathcal{B})$ (for example, when $s \geq 3$ ), a mild solution will be in fact a classical one.

Using (3.4), (3.5), and the boundedness of $\mathcal{P}^{-1}, \alpha(D), R_{1}, R_{2}$ again, one can check that $\mathcal{F}(0)=0$ and that there is a constant $C$ for which $\| \mathcal{F}(U)-$ $\mathcal{F}(\tilde{U})\left\|_{s} \leq C R\right\| U-\tilde{U} \|_{s}$ whenever $U=\left(\mu_{1}, \nu_{1}, \nu_{2}\right)^{T}$ and $\tilde{U}=\left(\tilde{\mu}_{1}, \tilde{\nu}_{1}, \tilde{\nu}_{2}\right)^{T}$ are selected from the closed ball $\bar{B}_{R}$ of radius $R$ centred at 0 in $X_{T}^{s}$. For fixed $U_{0} \in H^{s}\left(\mathbb{R}^{2}\right)^{3}$, as in the proof of Theorem 3.1, one can show that the mapping $\tilde{U} \mapsto U$, where

$$
U(t)=S(t) U_{0}+\int_{0}^{t} S(t-\tau) \mathcal{F}(\tilde{U}) d \tau,
$$

is a contraction of $\bar{B}_{R}$ into itself for $R$ sufficiently large and $T$ sufficiently small. Theorem 3.2 then follows immediately if we transform back to the original variable $\left(\zeta, v_{1}, v_{2}\right)$.

REMARK 3.1. Because the solution is given as the fixed point of a contraction mapping, one can prove that the correspondence $\left(\zeta_{0}, V_{0}\right) \mapsto(\zeta, V)$, between the initial data and the associated solution, is locally Lipschitz continuous.

REMARK 3.2. It is clear that the well-posedness results in Section 3.1 are also true in the case of one dimension because the method of the proof works for both one and two dimensions. It is worth noticing that if instead of (3.3), we use the inequality

$$
\|f g\|_{s-1} \leq\|f\|_{s}\|g\|_{s}, \quad s \geq 0,
$$

which is only valid in one dimension (see e.g. [9] for a proof), then one can take $s \geq 0$ in Theorems 3.1 and 3.2 in the case of one dimension. Thus, the 
well-posedness results in the one-dimensional case are slightly better than in two dimensions.

3.2. Other admissible Boussinesq/Boussinesq systems. In this section, we investigate the admissible cases not covered in Section 3.1, i.e. when

$$
a_{2}=0 \quad \text { or } \quad a_{4}=0 .
$$

They are classified according to the order of $\alpha(\mathbf{k})$ defined in Section 3.1. Recall that

$$
\alpha(\mathbf{k})=\left[\frac{\left(\frac{1}{\gamma+\delta}-\mu a_{1}|\mathbf{k}|^{2}\right)\left(1+\mu a_{4}|\mathbf{k}|^{2}\right)}{\left[(1-\gamma)\left(1-\mu a_{3}|\mathbf{k}|^{2}\right)+\varepsilon \sqrt{\mu} \nu|\mathbf{k}|^{2}\right]\left(1+\mu a_{2}|\mathbf{k}|^{2}\right)}\right]^{1 / 2} .
$$

Thus,

- $\alpha(\mathbf{k})$ is of order 2 when $a_{2}=0, a_{4}>0, a_{1}<0, a_{3}=\varepsilon \nu /((1-\gamma) \sqrt{\mu})$.

- $\alpha(\mathbf{k})$ is of order 1 when $a_{2}=0, a_{4}>0, a_{1}<0, a_{3}<\varepsilon \nu /((1-\gamma) \sqrt{\mu})$.

- $\alpha(\mathbf{k})$ is of order 0 when $a_{2}>0, a_{4}=0, a_{1}<0, a_{3}=\varepsilon \nu /((1-\gamma) \sqrt{\mu})$ or $a_{2}=0, a_{4}>0, a_{1}=0, a_{3}<\varepsilon \nu /((1-\gamma) \sqrt{\mu})$.

- $\alpha(\mathbf{k})$ is of order -1 when $a_{2}>0, a_{4}=0, a_{1}<0, a_{3}<\varepsilon \nu /((1-\gamma) \sqrt{\mu})$ or $a_{2}>0, a_{4}=a_{1}=0, a_{3}=\varepsilon \nu /((1-\gamma) \sqrt{\mu})$.

In these cases, we will use energy methods to study the local wellposedness of the Cauchy problem for the Boussinesq/Boussinesq systems (3.1). In order to estimate the nonlinear terms, we need the following estimates.

Lemma 3.2. Let $s \geq 0$.

(i) We have

$$
\left\|\Lambda^{s}(f g)\right\|_{0} \leq C\left(|f|_{\infty}\|g\|_{s}+\|f\|_{s}|g|_{\infty}\right) .
$$

(ii) $\left\|\left[\Lambda^{s}, f\right] g\right\|_{0} \leq C\left(|\nabla f|_{\infty}\|g\|_{s-1}+\|f\|_{s}|g|_{\infty}\right)$. In particular,

$$
\left\|\left[\Lambda^{s}, u\right] \nabla u\right\|_{0} \leq C|\nabla u|_{\infty}\|u\|_{s} .
$$

(iii) We have

$$
\left\|\left[\Lambda^{s}, f\right] . \nabla g\right\|_{0} \leq C\left(|\nabla f|_{\infty}\|g\|_{s}+\|f\|_{s+1}|g|_{\infty}\right) .
$$

Here $f$ and $g$ are assumed to be such that the right-hand side of the corresponding estimate is finite.

Proof. The first point is a classical estimate (see e.g. [18]). The second point is the classical Kato-Ponce estimate [25]. To prove the third point, we note that

$$
\begin{aligned}
{\left[\Lambda^{s}, f\right] . \nabla g } & =\Lambda^{s}(f . \nabla g)-f \cdot \Lambda^{s} \nabla g=\Lambda^{s} \nabla \cdot(f g)-f \cdot \Lambda^{s} \nabla g-\Lambda^{s}(\nabla . f g) \\
& =\left[\Lambda^{s} \nabla, f\right] g-\Lambda^{s}(\nabla . f g) .
\end{aligned}
$$


Using the Kato-Ponce estimate, we have

$$
\left\|\left[\Lambda^{s} \nabla, f\right] g\right\|_{0} \leq C\left(|\nabla f|_{\infty}\|g\|_{s}+\|f\|_{s+1}|g|_{\infty}\right) .
$$

On the other hand,

$$
\left\|\Lambda^{s}(\nabla \cdot f g)\right\|_{0} \leq C\left(|\nabla \cdot f|_{\infty}\|g\|_{s}+\|\nabla \cdot f\|_{s}|g|_{\infty}\right) .
$$

Combining the above two estimates yields the result.

3.2.1. Boussinesq/Boussinesq systems when $\alpha(\mathbf{k})$ has order 2. This subsection is concerned with cases where

$$
a_{2}=0, \quad a_{4}>0, \quad a_{1}<0, \quad a_{3}=\frac{\varepsilon \nu}{(1-\gamma) \sqrt{\mu}} .
$$

The system then has the form

$$
\left\{\begin{array}{l}
\partial_{t} \zeta+\frac{1}{\gamma+\delta} \nabla \cdot V+\varepsilon \frac{\delta^{2}-\gamma}{(\gamma+\delta)^{2}} \nabla \cdot(\zeta V) \\
\quad-\frac{\varepsilon \delta^{2}}{(\gamma+\delta)^{2}} \nabla \cdot(b V)+\mu a_{1} \Delta \nabla \cdot V=0, \\
\left(1-\mu a_{4} \Delta\right) \partial_{t} V+(1-\gamma) \nabla \zeta+\frac{\varepsilon}{2} \frac{\delta^{2}-\gamma}{(\gamma+\delta)^{2}} \nabla|V|^{2}=0 .
\end{array}\right.
$$

Define

$$
\begin{aligned}
X^{s+2}\left(\mathbb{R}^{d}\right)=\left\{V \in H^{s}\left(\mathbb{R}^{d}\right):\right. & \\
\|V\|_{X^{s+2}}^{2} & \left.=\left\|\Lambda^{s} V\right\|_{0}^{2}+\left\|\Lambda^{s} \nabla V\right\|_{0}^{2}+\left\|\Lambda^{s} \Delta V\right\|_{0}<\infty\right\} .
\end{aligned}
$$

Note that $X^{s+2}\left(\mathbb{R}^{d}\right)=H^{s+2}\left(\mathbb{R}^{d}\right)$ when $d=1$.

We now prove the following theorem.

TheOREM 3.3. Let $s>1$ and $b \in H^{s+1}\left(\mathbb{R}^{2}\right),\left(\zeta_{0}, V_{0}\right) \in H^{s}\left(\mathbb{R}^{2}\right) \times$ $X^{s+2}\left(\mathbb{R}^{2}\right)^{2}$. Then there exist $T>0$ and a unique solution

$$
(\zeta, V) \in X_{T}=C\left([0, T] ; H^{s}\left(\mathbb{R}^{2}\right)\right) \times C\left([0, T] ; X^{s+2}\left(\mathbb{R}^{2}\right)\right)^{2}
$$

of (3.12) with initial condition $\left(\zeta_{0}, V_{0}\right)$.

Proof. (i) Existence. The first step is to derive formally a priori estimates on solutions of (3.12). Apply $\Lambda^{s}:=(1-\Delta)^{s / 2}$ to the two equations in the above system, then multiply the first equation by $(1-\gamma) \Lambda^{s} \zeta$, the second equation by $\frac{1}{\gamma+\delta} \Lambda^{s} V+\mu a_{1} \Lambda^{s} \Delta V$ and integrate over $\mathbb{R}^{2}$. By adding 
the resulting two equations, we obtain

$$
\begin{aligned}
\frac{1}{2} \frac{d}{d t} \int_{\mathbb{R}^{2}}\left[(1-\gamma)\left|\Lambda^{s} \zeta\right|^{2}+\frac{1}{\gamma+\delta}\left|\Lambda^{s} V\right|^{2}+\frac{1}{\gamma+\delta} \mu a_{4}\left|\Lambda^{s} \nabla V\right|^{2}\right. & \left.-\mu^{2} a_{1} a_{4}\left|\Lambda^{s} \Delta V\right|^{2}\right] d X \\
= & -\int_{\mathbb{R}^{2}}\left[(1-\gamma) \varepsilon \frac{\delta^{2}-\gamma}{(\gamma+\delta)^{2}} \Lambda^{s} \zeta \Lambda^{s} \nabla \cdot(\zeta V)-(1-\gamma) \frac{\varepsilon \delta^{2}}{(\gamma+\delta)^{2}} \Lambda^{s} \zeta \Lambda^{s} \nabla \cdot(b V)\right. \\
& \left.+\frac{\varepsilon}{2} \frac{\delta^{2}-\gamma}{(\gamma+\delta)^{3}} \Lambda^{s} V \Lambda^{s} \nabla|V|^{2}+\frac{\varepsilon}{2} \frac{\delta^{2}-\gamma}{(\gamma+\delta)^{2}} \mu a_{1} \Lambda^{s} \Delta V \Lambda^{s} \nabla|V|^{2}\right] d X .
\end{aligned}
$$

We now estimate the terms on the right-hand side of the above equality. For the first term, we have

$$
\begin{aligned}
&\left|\left\langle\Lambda^{s} \zeta, \Lambda^{s} \nabla \cdot(\zeta V)\right\rangle\right|=\left|\left\langle\Lambda^{s}(V . \nabla \zeta), \Lambda^{s} \zeta\right\rangle+\left\langle\Lambda^{s}(\zeta \nabla \cdot V), \Lambda^{s} \zeta\right\rangle\right| \\
&=\left|\left\langle\left[\Lambda^{s}, V\right] \cdot \nabla \zeta, \Lambda^{s} \zeta\right\rangle+\left\langle V \cdot \Lambda^{s} \nabla \zeta, \Lambda^{s} \zeta\right\rangle+\left\langle\Lambda^{s}(\zeta \nabla \cdot V), \Lambda^{s} \zeta\right\rangle\right| \\
&=\left|\left[\Lambda^{s}, V\right] \cdot \nabla \zeta, \Lambda^{s} \zeta-\frac{1}{2}\left\langle\nabla \cdot V,\left|\Lambda^{s} \zeta\right|^{2}\right\rangle+\left\langle\Lambda^{s}(\zeta \nabla \cdot V), \Lambda^{s} \zeta\right\rangle\right| \\
& \leq\left\|\left[\Lambda^{s}, V\right] \cdot \nabla \zeta\right\|_{0}\left\|\Lambda^{s} \zeta\right\|_{0}+\frac{1}{2}|\nabla \cdot V|_{\infty}\left\|\Lambda^{s} \zeta\right\|_{0}^{2}+\left\|\Lambda^{s}(\zeta \nabla \cdot V)\right\|_{0}\left\|\Lambda^{s} \zeta\right\|_{0} \\
& \leq C\left(\left(|\nabla V|_{\infty}\|\zeta\|_{s}+\|V\|_{s+1}|\zeta|_{\infty}\right)\|\zeta\|_{s}+|\nabla \cdot V|_{\infty}\|\zeta\|_{s}^{2}\right. \\
&\left.+\left(|\zeta|_{\infty}\|\nabla \cdot V\|_{s}+\|\zeta\|_{s}|\nabla \cdot V|_{\infty}\right)\|\zeta\|_{s}\right) \\
& \leq C\left(\|\zeta\|_{s}^{3}+\|V\|_{X^{s+2}}^{3}\right),
\end{aligned}
$$

where we have used (3.11), (3.9), the fact that $H^{s}\left(\mathbb{R}^{2}\right) \hookrightarrow L^{\infty}\left(\mathbb{R}^{2}\right)$ for $s>1$, and Young's inequality $\left(a b \leq a^{p} / p+a^{q} / q, 1 / p+1 / q=1\right)$. Similarly, we estimate the second term:

$$
\left|\left\langle\Lambda^{s} \zeta, \Lambda^{s} \nabla \cdot(b V)\right\rangle\right| \leq C\left(\|\zeta\|_{s}^{2}+\|V\|_{X^{s+2}}^{2}\right),
$$

where the constant $C$ depends on $\|b\|_{s+1}$. In order to estimate the third and fourth terms, note that for a real-valued function $u$, we have the following estimates thanks to $(3.9)$ :

$$
\begin{aligned}
\left|\left\langle\Lambda^{s} u, \Lambda^{s} \partial_{x}\left(u^{2}\right)\right\rangle\right| & \leq 2\left\|\Lambda^{s} u\right\|_{0}\left\|\Lambda^{s}\left(u u_{x}\right)\right\|_{0} \\
& \leq C\|u\|_{s}\left(|u|_{\infty}\left\|u_{x}\right\|_{s}+\|u\|_{s}\left|u_{x}\right|_{\infty}\right), \\
\left|\left\langle\Lambda^{s} u_{x x}, \Lambda^{s} \partial_{x}\left(u^{2}\right)\right\rangle\right| & \leq 2\left\|\Lambda^{s} u_{x x}\right\|_{0}\left\|\Lambda^{s}\left(u u_{x}\right)\right\|_{0} \\
& \leq C\|u\|_{s+2}\left(|u|_{\infty}\left\|u_{x}\right\|_{s}+\|u\|_{s}\left|u_{x}\right|_{\infty}\right) .
\end{aligned}
$$

Similar estimates hold for $\left|\left\langle\Lambda^{s} u, \Lambda^{s} \partial_{y}\left(u^{2}\right)\right\rangle\right|$ and $\left|\left\langle\Lambda^{s} u_{y y}, \Lambda^{s} \partial_{y}\left(u^{2}\right)\right\rangle\right|$. Therefore, we have

$$
\left|\left\langle\Lambda^{s} V, \Lambda^{s} \nabla|V|^{2}\right\rangle\right| \leq C\|V\|_{s+1}^{3}, \quad\left|\left\langle\Lambda^{s} \Delta V, \Lambda^{s} \nabla|V|^{2}\right\rangle\right| \leq C\|V\|_{X^{s+2}}^{3} .
$$


Putting

$$
\begin{aligned}
Y(t)=\int_{\mathbb{R}^{2}}\left[(1-\gamma)\left|\Lambda^{s} \zeta\right|^{2}\right. & +\frac{1}{\gamma+\delta}\left|\Lambda^{s} V\right|^{2} \\
& \left.+\frac{1}{\gamma+\delta} \mu a_{4}\left|\Lambda^{s} \nabla V\right|^{2}-\mu^{2} a_{1} a_{4}\left|\Lambda^{s} \Delta V\right|^{2}\right] d X .
\end{aligned}
$$

Combining the above estimates, we get

$$
Y^{\prime}(t) \leq C_{1} Y(t)^{3 / 2}+C_{2} Y(t)
$$

which yields the bound of $Y(t)$ on $[0, T]$ for $T$ sufficiently small. Since $a_{1}<0$, $a_{4}>0$, we thus obtain the desired bound of $(\zeta, V)$ in $L^{\infty}\left(0, T ; H^{s}\left(\mathbb{R}^{2}\right)\right) \times$ $L^{\infty}\left(0, T ; X^{s+2}\left(\mathbb{R}^{2}\right)\right)^{2}$.

The next step is to justify the a priori estimate by regularizing the first equation in (3.12) with the dispersive regularizing term $-\varepsilon \Delta \partial_{t} \zeta$. The existence of a sequence of smooth approximate solutions $\left(\zeta_{\varepsilon}, V_{\varepsilon}\right)$ is already obtained in Theorem 3.2. By exactly the same procedure, one obtains a local bound on $\left(\zeta_{\varepsilon}, V_{\varepsilon}\right)$ in the space $L^{\infty}\left(0, T ; H^{s}\left(\mathbb{R}^{2}\right)\right) \times L^{\infty}\left(0, T ; X^{s+2}\left(\mathbb{R}^{2}\right)\right)^{2}$, where $T>0$ does not depend on $\varepsilon>0$ (see [9, p. 936] for a similar and more detailed argument). A standard application of the compactness method (see [28, Chapter 1]) yields the existence of a solution $(\zeta, V)$ in the space $L^{\infty}\left(0, T ; H^{s}\left(\mathbb{R}^{2}\right)\right) \times L^{\infty}\left(0, T ; X^{s+2}\left(\mathbb{R}^{2}\right)\right)^{2}$. If, instead, one passes to the strong limit by using the Bona-Smith technique [13], one deduces that, in fact, the solution $(\zeta, V)$ lies in $C\left([0, T] ; H^{s}\left(\mathbb{R}^{2}\right)\right) \times C\left([0, T] ; X^{s+2}\left(\mathbb{R}^{2}\right)\right)^{2}$.

(ii) Uniqueness. Let $\left(\zeta_{1}, V_{1}\right),\left(\zeta_{2}, V_{2}\right)$ be two solutions of (3.12) in the space $C\left([0, T] ; H^{s}\left(\mathbb{R}^{2}\right)\right) \times C\left([0, T] ; X^{s+2}\left(\mathbb{R}^{2}\right)\right)^{2}$ with the same value at $t=0$. Put $\zeta=\zeta_{1}-\zeta_{2}$ and $V=V_{1}-V_{2}$. Then $(\zeta, V)$ satisfies the system

$$
\left\{\begin{array}{l}
\partial_{t} \zeta+\frac{1}{\gamma+\delta} \nabla \cdot V+\varepsilon \frac{\delta^{2}-\gamma}{(\gamma+\delta)^{2}}\left[\nabla \cdot\left(\zeta_{1} V_{1}\right)-\nabla \cdot\left(\zeta_{2} V_{2}\right)\right] \\
\quad-\frac{\varepsilon \delta^{2}}{(\gamma+\delta)^{2}} \nabla \cdot(b V)+\mu a_{1} \Delta \nabla \cdot V=0, \\
\left(1-\mu a_{4} \Delta\right) \partial_{t} V+(1-\gamma) \nabla \zeta+\frac{\varepsilon}{2} \frac{\delta^{2}-\gamma}{(\gamma+\delta)^{2}}\left(\nabla\left|V_{1}\right|^{2}-\nabla\left|V_{2}\right|^{2}\right)=0,
\end{array}\right.
$$

with zero initial data. Repeating the argument used in part (i), we obtain

$$
\begin{aligned}
\frac{1}{2} \frac{d}{d t} \int_{\mathbb{R}^{2}}\left[(1-\gamma)\left|\Lambda^{s} \zeta\right|^{2}+\right. & \frac{1}{\gamma+\delta}\left|\Lambda^{s} V\right|^{2} \\
& \left.+\frac{1}{\gamma+\delta} \mu a_{4}\left|\Lambda^{s} \nabla V\right|^{2}-\mu^{2} a_{1} a_{4}\left|\Lambda^{s} \Delta V\right|^{2}\right] d X
\end{aligned}
$$




$$
\begin{aligned}
= & -\int_{\mathbb{R}^{2}}\left[(1-\gamma) \varepsilon \frac{\delta^{2}-\gamma}{(\gamma+\delta)^{2}} \Lambda^{s} \zeta \Lambda^{s}\left[\nabla \cdot\left(\zeta_{1} V_{1}\right)-\nabla \cdot\left(\zeta_{2} V_{2}\right)\right]\right. \\
& -(1-\gamma) \frac{\varepsilon \delta^{2}}{(\gamma+\delta)^{2}} \Lambda^{s} \zeta \Lambda^{s} \nabla \cdot(b V) \\
& +\frac{\varepsilon}{2} \frac{\delta^{2}-\gamma}{(\gamma+\delta)^{3}} \Lambda^{s} V \Lambda^{s}\left(\nabla\left|V_{1}\right|^{2}-\nabla\left|V_{2}\right|^{2}\right) \\
& \left.+\frac{\varepsilon}{2} \frac{\delta^{2}-\gamma}{(\gamma+\delta)^{2}} \mu a_{1} \Lambda^{s} \Delta V \Lambda^{s}\left(\nabla\left|V_{1}\right|^{2}-\nabla\left|V_{2}\right|^{2}\right)\right] d X .
\end{aligned}
$$

Observe that

$$
\begin{aligned}
\nabla \cdot\left(\zeta_{1} V_{1}\right)-\nabla \cdot\left(\zeta_{2} V_{2}\right) & =\nabla \zeta \cdot V_{1}+\zeta \nabla \cdot V_{1}+\nabla \zeta_{2} \cdot V+\zeta_{2} \nabla \cdot V, \\
\nabla\left|V_{1}\right|^{2}-\nabla\left|V_{2}\right|^{2} & =\nabla\left(V \cdot V_{1}\right)+\nabla\left(V . V_{2}\right) .
\end{aligned}
$$

By calculations similar to those appearing in the derivation of the a priori estimate, we find that

$$
Y^{\prime}(t) \leq C Y(t)
$$

where the constant $C$ depends on $\varepsilon, \gamma, \delta,\|b\|_{s+1},\left\|\zeta_{i}\right\|_{s},\left\|V_{i}\right\|_{X^{s+2}}(i=1,2)$, and $Y(t)$ is defined in part (i). Since $Y(0)=0$, the uniqueness follows immediately from Gronwall's inequality.

REMARK 3.3. In the proof of the theorems below, we will only establish a priori estimates of solutions. Then the existence and uniqueness of solutions can be obtained by adding a suitable dispersive regularizing term, and proceeding as in the proof of Theorem 3.3.

3.2.2. Boussinesq/Boussinesq systems when $\alpha(\mathbf{k})$ has order 1 . This subsection is concerned with cases where

$$
a_{2}=0, \quad a_{4}>0, \quad a_{1}<0, \quad a_{3}<\frac{\varepsilon \nu}{(1-\gamma) \sqrt{\mu}} .
$$

The system then has the form

$$
\left\{\begin{array}{c}
\partial_{t} \zeta+\frac{1}{\gamma+\delta} \nabla \cdot V+\varepsilon \frac{\delta^{2}-\gamma}{(\gamma+\delta)^{2}} \nabla \cdot(\zeta V) \\
\quad-\frac{\varepsilon \delta^{2}}{(\gamma+\delta)^{2}} \nabla \cdot(b V)+\mu a_{1} \Delta \nabla \cdot V=0 \\
\left(1-\mu a_{4} \Delta\right) \partial_{t} V+(1-\gamma) \nabla \zeta+\frac{\varepsilon}{2} \frac{\delta^{2}-\gamma}{(\gamma+\delta)^{2}} \nabla|V|^{2} \\
+\mu a_{3}(1-\gamma) \Delta \nabla \zeta-\varepsilon \sqrt{\mu} \nu \Delta \nabla \zeta=0
\end{array}\right.
$$

We now prove the following theorem.

ThEOREM 3.4. Let $s>1$ and $b \in H^{s+1}\left(\mathbb{R}^{2}\right),\left(\zeta_{0}, V_{0}\right) \in H^{s}\left(\mathbb{R}^{2}\right) \times$ $H^{s+1}\left(\mathbb{R}^{2}\right)^{2}$. Then there exist $T>0$ and a unique solution

$$
(\zeta, V) \in X_{T}=C\left([0, T] ; H^{s}\left(\mathbb{R}^{2}\right)\right) \times C\left([0, T] ; H^{s+1}\left(\mathbb{R}^{2}\right)\right)^{2}
$$

of $(3.13)$ with initial condition $\left(\zeta_{0}, V_{0}\right)$. 
Proof. We content ourselves with providing the proof of an a priori estimate that may be justified just as in Section 3.2.1 by regularizing the first equation in (3.13) with the term $-\varepsilon \Delta \partial_{t} \zeta$. As we already have a theory in this case given by Theorem 3.2, it is simply a matter of allowing $\varepsilon$ tend to zero using the Bona-Smith technique [13].

Applying $\Lambda^{s}$ to the two equations in the above system, then taking the $L^{2}$ scalar product of the first equation with $\left[-\mu a_{3}(1-\gamma)+\varepsilon \sqrt{\mu} \nu\right] \Lambda^{s} \zeta$, the second equation with $-\mu a_{1} \Lambda^{s} V$, and adding the resulting two equations, we obtain

$$
\begin{aligned}
\frac{1}{2} \frac{d}{d t} \int_{\mathbb{R}^{2}}\left[\left[-\mu a_{3}(1-\gamma)+\varepsilon \sqrt{\mu} \nu\right]\left|\Lambda^{s} \zeta\right|^{2}-\mu a_{1}\left|\Lambda^{s} V\right|^{2}-\mu^{2} a_{1} a_{4}\left|\Lambda^{s} \nabla V\right|^{2}\right] d X \\
=\int_{\mathbb{R}^{2}}\left[\frac{1}{\gamma+\delta}\left[\mu a_{3}(1-\gamma)-\varepsilon \sqrt{\mu} \nu\right] \Lambda^{s} \zeta \Lambda^{s} \nabla \cdot V\right. \\
\quad+\varepsilon \frac{\delta^{2}-\gamma}{(\gamma+\delta)^{2}}\left[\mu a_{3}(1-\gamma)-\varepsilon \sqrt{\mu} \nu\right] \Lambda^{s} \zeta \Lambda^{s} \nabla \cdot(\zeta V) \\
\quad-\frac{\varepsilon \delta^{2}}{(\gamma+\delta)^{2}}\left[\mu a_{3}(1-\gamma)-\varepsilon \sqrt{\mu} \nu\right] \Lambda^{s} \zeta \Lambda^{s} \nabla \cdot(b V)+(1-\gamma) \mu a_{1} \Lambda^{s} V \Lambda^{s} \nabla \zeta \\
\left.\quad+\frac{\varepsilon}{2} \frac{\delta^{2}-\gamma}{(\gamma+\delta)^{2}} \mu a_{1} \Lambda^{s} V \Lambda^{s} \nabla|V|^{2}\right] d X .
\end{aligned}
$$

We estimate the first and second terms on the right-hand side of the above equality as follows:

$$
\begin{aligned}
\left|\left\langle\Lambda^{s} \zeta, \Lambda^{s} \nabla \cdot V\right\rangle\right| & \leq\left\|\Lambda^{s} \zeta\right\|_{0}\left\|\Lambda^{s} \nabla \cdot V\right\|_{0}=\|\zeta\|_{s}\|\nabla \cdot V\|_{s} \leq\|\zeta\|_{s}\|V\|_{s+1}, \\
\left|\left\langle\Lambda^{s} V, \Lambda^{s} \nabla \zeta\right\rangle\right| & =\left|-\left\langle\Lambda^{s} \nabla \cdot V, \Lambda^{s} \zeta\right\rangle\right| \leq\left\|\Lambda^{s} \nabla \cdot V\right\|_{0}\left\|\Lambda^{s} \zeta\right\|_{0} \leq\|V\|_{s+1}\|\zeta\|_{s} .
\end{aligned}
$$

Other terms can be estimated exactly as in the proof of Theorem 3.3:

$$
\begin{aligned}
\left|\left\langle\Lambda^{s} \zeta, \Lambda^{s} \nabla \cdot(\zeta V)\right\rangle\right| & \leq C\left(\|\zeta\|_{s}^{3}+\|V\|_{s+1}^{3}\right), \\
\left|\left\langle\Lambda^{s} \zeta, \Lambda^{s} \nabla \cdot(b V)\right\rangle\right| & \leq C\left(\|\zeta\|_{s}^{2}+\|V\|_{s+1}^{2}\right), \\
\left|\left\langle\Lambda^{s} V, \Lambda^{s} \nabla|V|^{2}\right\rangle\right| & \leq C\|V\|_{s+1}^{3} .
\end{aligned}
$$

Setting

$$
Y(t)=\int_{\mathbb{R}^{2}}\left[\left[-\mu a_{3}(1-\gamma)+\varepsilon \sqrt{\mu} \nu\right]\left|\Lambda^{s} \zeta\right|^{2}-\mu a_{1}\left|\Lambda^{s} V\right|^{2}-\mu^{2} a_{1} a_{4}\left|\Lambda^{s} \nabla V\right|^{2}\right] d X,
$$

we deduce from the above estimates that

$$
Y^{\prime}(t) \leq C_{1} Y(t)^{3 / 2}+C_{2} Y(t)
$$

which provides a bound of $Y(t)$ on $[0, T]$ for $T$ sufficiently small. Since $a_{1}<0, a_{4}>0, a_{3}<\varepsilon \nu /((1-\gamma) \sqrt{\mu})$, we get the desired bound of $(\zeta, V)$ in $L^{\infty}\left(0, T ; H^{s}\left(\mathbb{R}^{2}\right)\right) \times L^{\infty}\left(0, T ; H^{s+1}\left(\mathbb{R}^{2}\right)\right)^{2}$. 
3.2.3. Boussinesq/Boussinesq systems when $\alpha(\mathbf{k})$ has order 0 . In this subsection, we consider systems in (3.1) with

$$
a_{2}>0, \quad a_{4}=0, \quad a_{1}<0, \quad a_{3}=\frac{\varepsilon \nu}{(1-\gamma) \sqrt{\mu}},
$$

and those with

$$
a_{2}=0, \quad a_{4}>0, \quad a_{1}=0, \quad a_{3}<\frac{\varepsilon \nu}{(1-\gamma) \sqrt{\mu}} .
$$

These two cases can both be studied using the techniques presented below. Therefore, the proof is only given for the first case. Then the system has the form

$$
\left\{\begin{array}{c}
\left(1-\mu a_{2} \Delta\right) \partial_{t} \zeta+\frac{1}{\gamma+\delta} \nabla \cdot V+\varepsilon \frac{\delta^{2}-\gamma}{(\gamma+\delta)^{2}} \nabla \cdot(\zeta V) \\
-\frac{\varepsilon \delta^{2}}{(\gamma+\delta)^{2}} \nabla \cdot(b V)+\mu a_{1} \Delta \nabla \cdot V=0 \\
\partial_{t} V+(1-\gamma) \nabla \zeta+\frac{\varepsilon}{2} \frac{\delta^{2}-\gamma}{(\gamma+\delta)^{2}} \nabla|V|^{2}=0 .
\end{array}\right.
$$

Theorem 3.5. Let $s>2$ and $b \in H^{s-1}\left(\mathbb{R}^{2}\right),\left(\zeta_{0}, V_{0}\right) \in H^{s}\left(\mathbb{R}^{2}\right)^{3}$. Then there exist $T>0$ and a unique solution

$$
(\zeta, V) \in X_{T}=C\left([0, T] ; H^{s}\left(\mathbb{R}^{2}\right)\right)^{3}
$$

of (3.14) with initial condition $\left(\zeta_{0}, V_{0}\right)$.

Proof. We only prove an a priori estimate; that may be justified just as in Section 3.2.1 by regularizing the second equation in (3.14) with the term $-\varepsilon \Delta \partial_{t} V$. form

First, consider the case $a_{1}=-\frac{1}{\gamma+\delta} a_{2}$. Then (3.14) may be given in the

$$
\left\{\begin{array}{l}
\partial_{t} \zeta+\frac{1}{\gamma+\delta} \nabla \cdot V+\left(1-\mu a_{2} \Delta\right)^{-1}\left[\varepsilon \frac{\delta^{2}-\gamma}{(\gamma+\delta)^{2}} \nabla \cdot(\zeta V)-\frac{\varepsilon \delta^{2}}{(\gamma+\delta)^{2}} \nabla \cdot(b V)\right]=0, \\
\partial_{t} V+(1-\gamma) \nabla \zeta+\frac{\varepsilon}{2} \frac{\delta^{2}-\gamma}{(\gamma+\delta)^{2}} \nabla|V|^{2}=0 .
\end{array}\right.
$$

Apply $\Lambda^{s}$ to the two equations in the above system, then multiply the first equation by $(1-\gamma) \Lambda^{s} \zeta$ and the second by $\frac{1}{\gamma+\delta} \Lambda^{s} V$ and integrate over $\mathbb{R}^{2}$. By adding the resulting two equations, we obtain 


$$
\begin{aligned}
& \frac{1}{2} \frac{d}{d t} \int_{\mathbb{R}^{2}}\left[(1-\gamma)\left|\Lambda^{s} \zeta\right|^{2}+\frac{1}{\gamma+\delta}\left|\Lambda^{s} V\right|^{2}\right] d X \\
&=-\int_{\mathbb{R}^{2}}\left[(1-\gamma) \varepsilon \frac{\delta^{2}-\gamma}{(\gamma+\delta)^{2}} \Lambda^{s} \zeta\left(1-\mu a_{2} \Delta\right)^{-1} \nabla \cdot \Lambda^{s}(\zeta V)\right. \\
&-(1-\gamma) \frac{\varepsilon \delta^{2}}{(\gamma+\delta)^{2}}\left(1-\mu a_{2} \Delta\right)^{-1} \nabla \cdot \Lambda^{s}(b V) \\
&\left.+\frac{1}{\gamma+\delta} \frac{\varepsilon}{2} \frac{\delta^{2}-\gamma}{(\gamma+\delta)^{2}} \Lambda^{s} V \Lambda^{s} \nabla|V|^{2}\right] d X .
\end{aligned}
$$

We now estimate the terms on the right-hand side of the above equality. First, we have

$$
\begin{array}{r}
\left|\left\langle\Lambda^{s} \zeta,\left(1-\mu a_{2} \Delta\right)^{-1} \nabla \cdot \Lambda^{s}(\zeta V)\right\rangle\right| \leq\left\|\Lambda^{s} \zeta\right\|_{0}\left\|\left(1-\mu a_{2} \Delta\right)^{-1} \nabla \cdot(\zeta V)\right\|_{s} \\
\leq C\|\zeta\|_{s}\|\zeta V\|_{s-1} \leq C\|\zeta\|_{s}\|\zeta\|_{s}\|V\|_{s} \leq C\left(\|\zeta\|_{s}^{3}+\|V\|_{s}^{3}\right),
\end{array}
$$

where we have used (3.4), (3.3) and Young's inequality. Similarly, we have

$$
\left|\left\langle\Lambda^{s} \zeta,\left(1-\mu a_{2} \Delta\right)^{-1} \nabla \cdot \Lambda^{s}(b V)\right\rangle\right| \leq C\left(\|\zeta\|_{s}^{2}+\|V\|_{s}^{2}\right),
$$

with $C$ depending on $\|b\|_{s-1}$. We now estimate the remaining term. Note that for a real-valued function $u$,

$$
\Lambda^{s} \partial_{x}\left(u^{2}\right)=2 \Lambda^{s}\left(u u_{x}\right)=2\left[\Lambda^{s}, u\right] u_{x}+2 u \Lambda^{s} u_{x} .
$$

Thus, using the Kato-Ponce estimate (3.10), we have

$$
\begin{aligned}
& \left|\left\langle\Lambda^{s} u, \Lambda^{s} \partial_{x}\left(u^{2}\right)\right\rangle\right|=\left|2\left\langle\Lambda^{s} u,\left[\Lambda^{s}, u\right] u_{x}\right\rangle+2\left\langle\Lambda^{s} u, u \Lambda^{s} u_{x}\right\rangle\right| \\
& \quad=\left|2\left\langle\Lambda^{s} u,\left[\Lambda^{s}, u\right] u_{x}\right\rangle+\left\langle u, \partial_{x}\left(\Lambda^{s} u\right)^{2}\right\rangle\right|=\left|2\left\langle\Lambda^{s} u,\left[\Lambda^{s}, u\right] u_{x}\right\rangle-\left\langle u_{x},\left(\Lambda^{s} u\right)^{2}\right\rangle\right| \\
& \quad \leq 2\left\|\Lambda^{s} u\right\|_{0}\left\|\left[\Lambda^{s}, u\right] u_{x}\right\|_{0}+\left|u_{x}\right|_{\infty}\left\|\Lambda^{s} u\right\|_{0}^{2} \leq C\left|u_{x}\right|_{\infty}\|u\|_{s}^{2} .
\end{aligned}
$$

A similar estimate holds for the term $\left|\left\langle\Lambda^{s} u, \Lambda^{s} \partial_{y}\left(u^{2}\right)\right\rangle\right|$. Thus, we have

$$
\left|\left\langle\Lambda^{s} V, \Lambda^{s} \nabla|V|^{2}\right\rangle\right| \leq C|\nabla V|_{\infty}\|V\|_{s}^{2} \leq\|V\|_{s}^{3} .
$$

Put

$$
Y(t)=\int_{\mathbb{R}^{2}}\left[(1-\gamma)\left|\Lambda^{s} \zeta\right|^{2}+\frac{1}{\gamma+\delta}\left|\Lambda^{s} V\right|^{2}\right] d X .
$$

From the above estimates, we get

$$
Y^{\prime}(t) \leq C_{1} Y(t)^{3 / 2}+C_{2} Y(t)
$$

which provides a bound of $Y(t)$ on $[0, T]$ for $T$ sufficiently small, and implies the desired bound of $(\zeta, V)$ in $L^{\infty}\left(0, T ; H^{s}\left(\mathbb{R}^{2}\right)\right)^{3}$.

In the general case, that is, (3.14) with $a_{1}<0, a_{2}>0$ and $a_{1} \neq-\frac{1}{\gamma+\delta} a_{2}$, observe that

$$
\frac{\frac{1}{\gamma+\delta}-\mu a_{1}|\mathbf{k}|^{2}}{1+\mu a_{2}|\mathbf{k}|^{2}}=-\frac{a_{1}}{a_{2}}+\frac{\frac{1}{\gamma+\delta}+\frac{a_{1}}{a_{2}}}{1+\mu a_{2}|\mathbf{k}|^{2}}
$$


so that (3.14) is equivalent to

$$
\left\{\begin{aligned}
\partial_{t} \zeta & -\frac{a_{1}}{a_{2}} \nabla \cdot V+\left(\frac{1}{\gamma+\delta}+\frac{a_{1}}{a_{2}}\right)\left(1-\mu a_{2} \Delta\right)^{-1} \nabla \cdot V \\
& +\left(1-\mu a_{2} \Delta\right)^{-1}\left[\varepsilon \frac{\delta^{2}-\gamma}{(\gamma+\delta)^{2}} \nabla \cdot(\zeta V)-\frac{\varepsilon \delta^{2}}{(\gamma+\delta)^{2}} \nabla \cdot(b V)\right]=0 \\
\partial_{t} V & +(1-\gamma) \nabla \zeta+\frac{\varepsilon}{2} \frac{\delta^{2}-\gamma}{(\gamma+\delta)^{2}} \nabla|V|^{2}=0 .
\end{aligned}\right.
$$

For this system, without any difficulty, we can repeat the above arguments to obtain an a priori estimate of $(\zeta, V)$ in $L^{\infty}\left(0, T ; H^{s}\left(\mathbb{R}^{2}\right)\right)^{3}$.

3.2.4. Boussinesq/Boussinesq systems when $\alpha(\mathbf{k})$ has order -1 . This corresponds to the cases where

$$
a_{2}>0, \quad a_{4}=0, \quad a_{1}<0, \quad a_{3}<\frac{\varepsilon \nu}{(1-\gamma) \sqrt{\mu}}
$$

or

$$
a_{2}>0, \quad a_{4}=0, \quad a_{1}=0, \quad a_{3}=\frac{\varepsilon \nu}{(1-\gamma) \sqrt{\mu}}
$$

These two cases can both be studied using the techniques presented below. We thus give the proof for the first case only. Then the system has the form

$$
\left\{\begin{array}{c}
\left(1-\mu a_{2} \Delta\right) \partial_{t} \zeta+\frac{1}{\gamma+\delta} \nabla \cdot V+\varepsilon \frac{\delta^{2}-\gamma}{(\gamma+\delta)^{2}} \nabla \cdot(\zeta V) \\
\quad-\frac{\varepsilon \delta^{2}}{(\gamma+\delta)^{2}} \nabla \cdot(b V)+\mu a_{1} \Delta \nabla \cdot V=0 \\
\partial_{t} V+(1-\gamma) \nabla \zeta+\frac{\varepsilon}{2} \frac{\delta^{2}-\gamma}{(\gamma+\delta)^{2}} \nabla|V|^{2} \\
+\mu a_{3}(1-\gamma) \Delta \nabla \zeta-\varepsilon \sqrt{\mu} \nu \Delta \nabla \zeta=0
\end{array}\right.
$$

THEOREM 3.6. Let $s>2$ and $b \in H^{s}\left(\mathbb{R}^{2}\right),\left(\zeta_{0}, V_{0}\right) \in H^{s+1}\left(\mathbb{R}^{2}\right) \times$ $H^{s}\left(\mathbb{R}^{2}\right)^{2}$. Then there exist $T>0$ and a unique solution

$$
(\zeta, V) \in X_{T}=C\left([0, T] ; H^{s+1}\left(\mathbb{R}^{2}\right)\right) \times C\left([0, T] ; H^{s}\left(\mathbb{R}^{2}\right)\right)^{2}
$$

of (3.15) with initial condition $\left(\zeta_{0}, V_{0}\right)$.

Proof. We only prove an a priori estimate. Apply $\Lambda^{s}$ to both equations in the above system, then multiply the first equation by $\left[-\mu a_{3}(1-\gamma)\right.$ $+\varepsilon \sqrt{\mu} \nu] \Lambda^{s} \zeta$, the second by $-\mu a_{1} \Lambda^{s} V$, and integrate over $\mathbb{R}^{2}$. By adding the resulting two equations and integrating by parts, we obtain 


$$
\begin{aligned}
& \frac{1}{2} \frac{d}{d t} \int_{\mathbb{R}^{2}}\left[\left[-\mu a_{3}(1-\gamma)+\varepsilon \sqrt{\mu} \nu\right]\left|\Lambda^{s} \zeta\right|^{2}\right. \\
& \left.\quad+\mu a_{2}\left[-\mu a_{3}(1-\gamma)+\varepsilon \sqrt{\mu} \nu\right]\left|\Lambda^{s} \nabla \zeta\right|^{2}-\mu a_{1}\left|\Lambda^{s} V\right|^{2}\right] d X \\
& =\int_{\mathbb{R}^{2}}\left[\frac{1}{\gamma+\delta}\left[-\mu a_{3}(1-\gamma)+\varepsilon \sqrt{\mu} \nu\right] \Lambda^{s} \nabla \zeta \cdot \Lambda^{s} V\right. \\
& \quad+\varepsilon \frac{\delta^{2}-\gamma}{(\gamma+\delta)^{2}}\left[-\mu a_{3}(1-\gamma)+\varepsilon \sqrt{\mu} \nu\right] \Lambda^{s} \nabla \zeta \cdot \Lambda^{s}(\zeta V) \\
& \quad-\frac{\varepsilon \delta^{2}}{(\gamma+\delta)^{2}}\left[-\mu a_{3}(1-\gamma)+\varepsilon \sqrt{\mu} \nu\right] \Lambda^{s} \nabla \zeta \cdot \Lambda^{s}(b V)-(1-\gamma) \mu a_{1} \Lambda^{s} V \Lambda^{s} \nabla \zeta \\
& \left.\quad-\frac{\varepsilon}{2} \frac{\delta^{2}-\gamma}{(\gamma+\delta)^{2}} \mu a_{1} \Lambda^{s} V \Lambda^{s} \nabla|V|^{2}\right] d X .
\end{aligned}
$$

The term $\left|\left\langle\Lambda^{s} V, \Lambda^{s} \nabla|V|^{2}\right\rangle\right|$ is estimated exactly as in the proof of Theorem 3.5. The other terms on the right-hand side can be estimated directly using Hölder's inequality and (3.9). As a result, one gets

$$
Y^{\prime}(t) \leq C_{1} Y(t)^{3 / 2}+C_{2} Y(t)
$$

where

$$
\begin{aligned}
& Y(t)=\int_{\mathbb{R}^{2}}\left[\left[-\mu a_{3}(1-\gamma)+\varepsilon \sqrt{\mu} \nu\right]\left|\Lambda^{s} \zeta\right|^{2}\right. \\
& \left.\quad+\mu a_{2}\left[-\mu a_{3}(1-\gamma)+\varepsilon \sqrt{\mu} \nu\right]\left|\Lambda^{s} \nabla \zeta\right|^{2}-\mu a_{1}\left|\Lambda^{s} V\right|^{2}\right] d X .
\end{aligned}
$$

This inequality implies a bound of $Y(t)$ on $[0, T]$ for $T$ sufficiently small. Since $a_{1}<0, a_{2}>0, a_{3}<\varepsilon \nu /((1-\gamma) \sqrt{\mu})$, it implies the desired bound of $(\zeta, V)$ in $L^{\infty}\left(0, T ; H^{s+1}\left(\mathbb{R}^{2}\right)\right) \times L^{\infty}\left(0, T ; H^{s}\left(\mathbb{R}^{2}\right)\right)^{2}$.

REMARK 3.4. It is clear that the energy methods, which are used to prove Theorems 3.3-3.6, also work in the case of one dimension. Moreover, noting that $H^{t}(\mathbb{R}) \hookrightarrow L^{\infty}(\mathbb{R})$ for $t>1 / 2$, we can take $s>1 / 2$ in Theorems 3.3 and 3.4, and $s>3 / 2$ in Theorems 3.5 and 3.6, in the case of one dimension. Thus, the well-posedness results in the one-dimensional case are better than in two dimensions as far as the assumed level of regularity of the initial data is concerned.

REMARK 3.5. When $\gamma=0, \delta=1$, we formally recover the Boussinesq systems for surface waves from the Boussinesq/Boussinesq systems (3.1). Thus, from the above theorems, we also obtain the well-posedness results to the Boussinesq systems (1.1). We leave the formulations of these results to the readers. In particular, the results of [9] extend to the case of two dimensions and to the case with surface tension. It is worth noticing that even in the case of one dimension, the results here (given by Remark 3.4) 
are better than the ones in [9], as far as the assumed level of regularity of the initial data is concerned (cf. Remark 3.13 in [9]).

Acknowledgements. This work was done while the author was visiting the Department of Mathematics of Paris-Sud University as a post-doctoral fellow. The author would like to thank Professor Jean-Claude Saut for helpful discussions. He would also like to acknowledge the hospitality and support of the Department.

The author thanks the referee for the helpful comments and suggestions which improved the presentation of the paper.

This work was supported by the National Foundation for Science and Technology Development (NAFOSTED).

\section{References}

[1] B. Alvarez-Samaniego and D. Lannes, Large time existence for 3D water-waves and asymptotics, Invent. Math. 171 (2008), 485-541.

[2] D. M. Ambrose, Well-posedness of vortex sheets with surface tension, SIAM J. Math. Anal. 35 (2003), 211-244.

[3] D. M. Ambrose and N. Masmoudi, Well-posedness of 3-D vortex sheets with surface tension, Comm. Math. Sci. 5 (2007), 391-430.

[4] C. J. Amick, Regularity and uniqueness of solutions to the Boussinesq system of equations, J. Differential Equations 54 (1984), 231-247.

[5] J. Angulo, On the Cauchy problem for a Boussinesq-type system, Adv. Differential Equations 4 (1999), 457-492.

[6] R. Barros, S. L. Gavrilov and V. M. Teshukov, Dispersive nonlinear waves in twolayer flows with free surface. I. Model derivation and general properties, Stud. Appl. Math. 119 (2007), 191-211.

[7] J. L. Bona and M. Chen, A Boussinesq system for two-way propagation of nonlinear dispersive waves, Phys. D 116 (1998), 191-224.

[8] J. L. Bona, M. Chen and J.-C. Saut, Boussinesq equations and other systems for small-amplitude long waves in nonlinear dispersive media. Part I. Derivation and linear theory, J. Nonlinear Sci. 12 (2002), 283-318.

[9] - - - - Boussinesq equations and other systems for small-amplitude long waves in nonlinear dispersive media. Part II. Nonlinear theory, Nonlinearity 17 (2004), 925-952.

[10] J. L. Bona, T. Colin and D. Lannes, Long wave aproximations for water-waves, Arch. Ration. Mech. Anal. 178 (2005), 373-410.

[11] J. L. Bona and H. Kalisch, Models for the internal waves in deep water, Discrete Contin. Dynam. Systems 6 (2000), 1-20.

[12] J. L. Bona, D. Lannes and J.-C. Saut, Asymptotic models for internal waves, J. Math. Pures Appl. (9) 89 (2008), 538-566.

[13] J. L. Bona and R. Smith, The initial-value problem for the Korteweg-de Vries equation, Philos. Trans. Roy. Soc. London A 278 (1975), 555-601.

[14] R. Camassa, W. Choi, H. Michallet, P.-O. Rusas and J. K. Sveen, On the realm of validity of strongly nonlinear asymptotic approximations for internal waves, J. Fluid Mech. 549 (2006), 1-23. 
[15] F. Chazel, Influence of bottom topography on water-waves in the long wave regime, Math. Model. Numer. Anal. 41 (2007), 771-799.

[16] W. Choi and R. Camassa, Weakly nonlinear internal waves in a two-fluid system, J. Fluid Mech. 313 (1996), 83-103.

[17] —, - Fully nonlinear internal waves in a two-fluid system, ibid. 396 (1999), 1-36.

[18] R. R. Coifman and Y. Meyer, Nonlinear harmonic analysis, operator theory and PDE, in: Beijing Lectures in Harmonic Analysis (Beijing, 1984), Ann. of Math. Stud. 112, Princeton Univ. Press, 3-45.

[19] W. Craig, P. Guyenne and H. Kalisch, Hamiltonian long-wave expansions for free surfaces and interfaces, Comm. Pure Appl. Math. 58 (2005), 1587-1641.

[20] V. A. Dougalis, D. E. Mitsotakis and J.-C. Saut, On some Boussinesq systems in two space dimensions: Theory and numerical analysis, ESAIM Math. Model. Numer. Anal. 41 (2007), 825-854.

[21] A. S. Fokas and B. Pelloni, Boundary value problems for Boussinesq type systems, Math. Phys. Anal. Geom. 8 (2005), 59-96.

[22] P. Grisvard, Quelques propriétés des espaces de Sobolev, utiles dans l'étude des équations de Navier-Stokes (I), in: Problèmes d'évolutions, non linéaires, Séminaire de Nice (1974-1976).

[23] T. Iguchi, Two-phase problem for two-dimensional water waves of infinite depth, Math. Models Methods Appl. Sci. 6 (1997), 791-821.

[24] T. Iguchi, N. Tanaka and A. Tani, On the two-phase free boundary problem for two-dimensional water waves, Math. Ann. 309 (1997), 199-223.

[25] T. Kato and G. Ponce, Commutator estimates and the Euler and Navier-Stokes equations, Comm. Pure Appl. Math. 41 (1988), 891-907.

[26] N. Kita and J. Segata, Well-posedness for the Boussinesq-type system related to the water wave, Funkcial. Ekvac. 47 (2004), 329-350.

[27] D. Lannes, Well-posedness of the water-waves equations, J. Amer. Math. Soc. 18 (2005), 605-654.

[28] J.-L. Lions, Quelques méthodes de résolution des problèmes aux limites non linéaires, Dunod, Paris, 1969.

[29] H. Y. Nguyen and F. Dias, A Boussinesq system for two-wave propagation of interfacial waves, Phys. D 237 (2008), 2365-2389.

[30] K. Ohi and T. Iguchi, A two-phase problem for capillary-gravity waves and the Benjamin-Ono equation, Discrete Contin. Dynam. Systems 23 (2009), 1205-1240.

[31] A. Pazy, Semigroups of Linear Operators and Applications to Partial Differential Equations, Springer, 1983.

[32] J. Shatah and C. Zeng, A priori estimates for the fluid interface problem, Comm. Pure Appl. Math. 61 (2008), 848-876.

[33] M. E. Schonbek, Existence of solutions for the Boussinesq system of equations, J. Differential Equations 42 (1981), 325-352.

Department of Mathematics

Hanoi University of Education

136 Xuan Thuy, Cau Giay, Hanoi, Vietnam

E-mail: anhctmath@hnue.edu.vn

Received 15.9.2008

and in final form 23.11.2008 
